\title{
Psychic tonus, body schema and the parietal lobes: A multiple lesion case analysis
}

\author{
C.M.J. Braun*, S. Desjardins, S. Gaudelet and A. Guimond \\ Centre de Neurosciences de la Cognition and Department of Psychology, Université du Québec à Montréal, Canada
}

\begin{abstract}
The psychic tonus model (Braun and colleagues, 1999, 2002, 2003, 2006) states that the left hemisphere is a "booster" of internal experience and behavior in general, and that the right hemisphere is a "dampener". Twenty-five patients with a "positive" extreme disturbance of body schema (somatoparaphrenia) and 37 patients with a "negative" disturbance of body schema (autotopagnosia or Gerstmann's syndrome), all following a unilateral parietal lesion, were found in the literature and were analyzed to test predictions from Braun's "psychic tonus" model. As expected, patients with a positive syndrome had a right hemisphere lesion significantly more frequently, and those with a negative syndrome had a left hemisphere lesion significantly more frequently. Thus the psychic tonus model of hemispheric specialization, previously supported with regard to psychomotor baseline, libido, talkativeness, memory, auditory and visual perceptual tonus, now incorporates the tonus of representation of the body (body schema) in the parietal lobes.
\end{abstract}

Keywords: Body schema, psychic tonus, hemispheric specialization, parietal lesions, hypergnosia, hypognosia

\section{Introduction}

\subsection{Psychic tonus: a hemispheric specialization interpretation of positive and negative symptoms}

Braun and colleagues (see Braun [1] for an in depth account) have developed a model of hemispheric specialization initially based on effects of focal lesions which they have termed "psychic tonus". This model proposes a new framework (but see Galin [2] for some premonitory ideas) for interpretation of positive and negative symptoms. The model draws equally from psychiatry as it does from neurology, as well as the biopsychology of normal and abnormal function in humans and animals. The model is based on demonstrations to the effect that mood [3], psychomotor baseline [3], libido [4], talkativeness [5], immune func-

*Address for correspondence: Claude M.J. Braun, PhD, Full professor, Centre de Neurosciences Cognitives UQAM, C.P 8888, Succ. Centre-Ville, Montréal, Québec, Canada, H3C 3P8. Tel.: +1 5149873000 (4814); Fax: +1 514987 8952; E-mail: Braun.Claude@UQAM.CA; URL: http://www.er.uqam.ca/nobel/ r31210/home.html. tion [6], memory [7], visual symptoms [8] and auditory symptoms [9] are all modulated in similar opposed ways by the two hemispheres, in right handers. The normal left hemisphere increases psychic tonus (infered from right hemisphere lesions), while the right decreases it (infered from left hemisphere lesions). In short, an organism with high psychic tonus will be mentally and behaviorally activated. Even the immune system is upregulated by left hemisphere activation. An organism with low psychic tonus will be all the contrary and will be characterized by right hemisphere activation.

The issue of whether perceptual function also falls under the orbit of hemispheric specialization for psychic tonus remains pending. Braun and colleagues [10] found that there were significantly more reports of cases of post lesion visual hallucination with right hemisphere lesions than left in the behavioral neurology litterature. It therefore appears reasonable to predict that post lesion perceptual anomalies should generally manifest a dissociation according to lesion side. Left hemisphere lesions should produce negative perceptual anomalies, a dulling of perceptual representation which we shall term hypognosia, while right hemisphere lesions should produce positive perceptual 
anomalies such as hallucinations, a lushness of perceptual representation, which we shall term hypergnosia. More prosaically, "positive" symptoms are understood here to consist of occurrence of undesired, unsollicited representations which ought not occur in a normal subject. We understand "negative" symptoms as inability to mobilize representations which should easily be mobilized at will by a normal person.

One problem with using neurological patients to test the veridicity of the "psychic tonus" concept is that extreme manifestations are required. We acknowledge that such cases are very rare, and that many cases, if not most, may carry bilateral lesions. Pure and extreme perceptual disorders resulting from well documented unilateral brain lesions are too rare to be assembled in a single analysis from any one clinical data base (neurology or psychiatry files of any one institution). That is why the exponentially increasing explosion of case reports today provides a data base fit for inference testing. The present report will limit itself to the somesthesic modality, and to cases with parietal lobe lesions exclusively.

Hemispheric specialization for somesthesic perception has been far less investigated than it has been for vision. Several authors have noted that hysterical somatic symptoms are more typically manifest on the left side of the body (see [11] for a review). This is compatible with the psychic tonus model but it is not the ideal test. As will be explained in the next sections, the ideal test of the psychic tonus model in the somesthesic modality would consist of determining the laterality of focal lesions causing a massively "positive" distorsion of body schema versus a massively "negative' distorsion. As far as we could determine, body schema has not been subjected to any such analysis crowned by inference tests of lesioned patients designed to test theoretical models of hemispheric specialization.

\subsection{Psychic tonus and "body schema"}

In their review of post lesion hallucinosis, Braun and colleagues (2003) observed that pure somesthesic hallucination tends to result from right parietal lesions, a finding which has been noted by several predecessors [12]. However, post lesion somesthesic hallucination has been too rare to support inference testing regarding its localisation. Likewise, pure post lesion autotopagnosia or loss of body schema has been noted to nearly always result from left parietal lesions $[13,14]$. The purpose of the present report was thus primarily to assemble an exhaustive review of single cases with ra- diological documentation of a focal unilateral parietal lesion leading either to a positive form of distorsion of "body schema" (bilateral somesthesic hallucinosis) or to a negative form (bilateral somesthesic agnosia).

\subsection{Disorders of body schema}

The term "body image" is often a misnomer in clinical neuroscience. Most clinical neuroscience authors, when talking about "body image" are not talking about body image at all, but rather about body schema, i.e., of somesthesic representation of one's own body rather than of visual representation. Though the term body image persists, we contend that it should be replaced by "body schema". Indeed, the congenitally blind have body schema, i.e., high level representation of body parts. It is significantly less precise than in normals, but it is far from autotopagnosia [15] and this is achieved despite complete absence of visual imagery, even from birth. So obviously visualisation need not be critical in higher order representation of the body. Also, estranged body schema is probably more easily expressed in "visual" terms by patients with expressions such as "I see my body split in two", "I see a third limb on myself", etc., when in probable fact, they "feel" those things somesthesically, and translate that feeling into expressions designed to be better understood by the interlocutor. Indeed, when a patient complains of a supernumerary limb, the first comments of the clinician are likely to be: "do you see it? show me where it is ... etc.". Suffice it to state that, for the purposes of the present report, we take the term "body schema" to designate a primarily somesthesic high level representation of the body.

The quintessential positive disorder of body schema is somatoparaphrenia [16]. When it is pure and is caused by a unilateral lesion, the localisation of the lesion is typically right parietal (as we shall explicitate in the results section). In the extreme form of somatoparaphrenia complex somesthesic hallucinations involve feelings of supernumerary or abnormally sized or deformed body parts, of feelings of being touched, or of involuntary movement involving both sides of the body. Other terms refering to less obviously "positive" anomalies of body schema include dysmorphobia (or body dysmorphic syndrome), autoscopia, micro and macrosomatognosia, phantom limb, auto-reduplication, self misidentification syndrome (reverse Capgras or Fregoli syndrome, see [17], somatisation disorder, and perhaps heautoscopy and autoscopia though the latter two are visual by definition. 
The quintessential and most extreme "negative" disorder of body schema is autotopagnosia [18]. It consists of a complete inability to localize or represent one's own body parts on both sides, a bilateral high level somesthesic agnosia, which often comprises bilateral anosagnosia as well. When it is pure and is caused by a unilateral lesion, the lesion is typically left parietal. Other terms refering to similar but less encompassing negative anomalies of body schema include Gerstmann's syndrome (especially the finger agnosia and right-left confusion elements), asymbolia for pain, anosagnosia, etc. Hécaen and Albert [19] considered Gerstmann's syndrome a mild form of autotopagnosia. Gerstmann's syndrome is relevant to the "psychic tonus" model primarily because one of its symptoms is an intrinsically bilateral distorsion of body schema", namely right-left confusion. We have found that when investigated for such, finger agnosia in Gerstmann's syndrome is also typically bilateral (see the results section), making that syndrome all the more relevant for the present report. Pure Gerstmann's syndrome is nearly always caused by a left parietal lesion. Since many cases of Gerstmann's syndrome have been published, this syndrome was also reviewed systematically for the present report.

\subsection{Unilateral neglect syndromes and the "psychic tonus" model}

There is overwhelming evidence in behavioral neurology to the effect that unilateral neglect, in any modality - including somesthesic (even with regard to representation of the body), results far more often from right than left lesions, and that the typical lesion producing hemineglect is inferior parietal. We believe that there is probably some truth to this, though exagerated. A pure test of tactile hemineglect must exclude any motor exploration component. Tactile extinction fits that requirement. Gainotti and colleagues [20] found that large cohorts of patients with right and left telencephalic lesions presented no lateralized difference in tactile extinction after correction for errors on the body side ipsilateral to the lesion. Schwartz and colleagues [21] obtained similar results with 274 patients with unilateral brain damage. This explains why many authors may have observed more tactile extinction after right hemisphere lesions: patients with massive left hemisphere lesions tend to get excluded from neuropsychological studies because they are too aphasic to understand elementary instructions, and/or left hemisphere lesioned patients present more bilateral neglect. In short, we believe the so-called "right hemisphere hemineglect" phenomenon is none other than a standard contralesional functional loss which is far less observed in left hemisphere cases simply because the neglect is bilateral in those cases. Nevertheless, the generally recognized propensity of right parietal lesions to produce hemineglect could be construed as the main objection (or perhaps the more exact word would be "challenge") which could be leveled, in the "perceptual domain", against the "psychic tonus" model of hemispheric specialization. Indeed, the psychic tonus model predicts negative cognitive-perceptuomotor symptoms after a left hemisphere lesion, rather than a right hemisphere lesion. Our answer to this is as follows. The dominant models of hemineglect are variants of what could be called the class of "subcortical mediation of orienting". Heilman and colleagues for example, propose a lateralized "corticolimbic reticular loop" and Kinsbourne proposes a "brainstem asymmetry", of lateral orientation [22]. These models insist on primitive pre-representational factors to the detriment of representational factors. One argument for this class of models is that hemineglect is often furtive: it tends to disappear spontaneously after a few weeks after the lesion - suggesting that it is due to a simple primitive mechanism that can be compensated for by representational feedback (the patient learns to invest extra attentional energy to the previously neglected side). Another argument for this class of models is that patients with left hemineglect are hyperoriented to the right side more than they are neglectful of the left side (they look more and they grope more on that side [23]). A third argument in support of this class of models is that subcortical lesions often produce hemineglect, including in the tactile modality [24].

The psychic tonus model has a wider angle than these diverse explanations of hemineglect. It proposes that representational neglect can be bilateral (e.g., autotopagnosia) following a unilateral telencephalic lesion of the left hemisphere, and that the same lesion located in the right hemisphere is more likely to produce the contrary syndrome, a syndrome of pathologically exuberant bilateral representation (e.g., somatoparaphrenia). Since we demonstrate that this is what typically occurs (see the results section), we are led to concur with authors who interpret hemineglect as a prerepresentational defect of orientation involving subcortical brain structures. Thus, lesions of the right inferior parietal lobe ought to produce somatoparaphrenia or related symptoms - as well as unilateral left hemineglect, and these two symptom complexes ought to be considered relatively independent - despite the fact 
that the lesion engendering them can be the same. We agree with Heilman and Kinsbourne that the parietal cortical lesion producing hemineglect probably causes that symptom via loss of connections to subcortical structures, but we add that somatoparaphrenia is a high level representational disorder probably caused directly by damage to parietal cortex. The psychic tonus model proposes that left parietal lesions ought to produce bilateral neglect (of which autotopagnosia is the severest form), without hallucinosis, and as stated above, that is exactly what most often occurs.

Many authors believe that somatoparaphrenia is nothing other than compensation for hemineglect [12, 25-28]. However, somatoparaphrenia is not always a hallucination of an extra body part on the left, it can be on the right body side or both sides or the body meridian, and hemiplegia and hemineglect are not necessarily present (see our results section). Somatoparaphrenia is clearly a "positive" syndrome because it typically consists of spontaneous and exuberant representation of something (e.g., a third limb) that is not in the "normal" repertoire.

\section{Method}

The selection criteria of the cases to be assembled were optimized to assure meaningful interpretation of localisation of brain damage in view of testing the "psychic tonus" model of hemispheric specialization with regard to "body schema". First, to assure that cases with right versus left pathology would be comparable and relevant, only etiologies involving the parietal lobes were considered. Careful attention was paid to lesion localization within the hemisphere to assure against a lesion size artefact. Several other possible intervening variables (age, gender, presence/absence of EEG, presence/absence of neurological exam, date of publication, presence/absence of aphasic symptoms, lesion etiology, presence/absence of hemineglect, psychiatric comorbidity, hemibody where the hallucination occurs) were also systematically tabulated for all cases.

\section{Results}

\subsection{Positive distorsions of body schema}

The first table reviews published cases of the most extreme form of positive distorsion of body schema resulting from a unilateral parietal lesion, with or without hemineglect. Cases with hemineglect are slightly less telling (for a test of the psychic tonus model of hemispheric specialization) than cases without hemineglect because there probably is indeed a relation between unilateral hallucinosis and unilateral hemineglect - consisting of compensation for sensory deprivation. Such cases present a mixture of positive and negative symptoms - to the extent that hemineglect can be construed as a "negative" symptom (though we think it should not be considered negative psychic tonus). We found only one case presenting both somatoparaphrenia and autotopagnosia and he was excluded because of the presence of both positive and negative body schema symptoms. Other less obviously "positive" bilateral higher order syndromes of distorted body schema (e.g., somatisation disorder, reverse Capgras syndrome) have been reported much less frequently to result from unilateral brain lesions. We found only two cases of reverse Capgras syndrome following a unilateral lesion, and though both had right hemisphere lesions, these were not parietal $[29,30]$. We found only three cases of somatisation disorder after a unilateral parietal lesion [31-33]. All three had a right hemisphere lesion. However, in the next table, we review only cases with somatoparaphrenia following a unilateral parietal lesion. See Table 1.

Of the 25 cases of somatoparaphrenia following a unilateral parietal lobe lesion of table 1,24 had a lesion on the right and one on the left (binomial: $p<0.0005$, two tailed).

We believe left and right hemisphere lesions have equiprobable chances of occuring and being selected for case reports (in the absence of selection for any type of mental disturbance). For example, Montour-Proulx and her colleagues [59] assembled 635 previously published post-lesion cases without selection for any psychiatric symptom. In their data base, 328 cases were left hemisphere damaged and 307 were right hemisphere damaged (binomial probability against equiprobabilty: $p>0.12$, two tailed).

\subsection{Negative distorsions of body schema}

The next table reviews published cases of the most prototypically negative and complete variant of distorsion of body schema, namely autotopagnosia -resulting from a unilateral parietal lesion. By definition, all these cases presented bilateral symptoms. Because several authors consider that autotopagnosia is a semantic aphasia [51], cases without aphasia represent a more interesting test of the psychic tonus model of hemi- 
Table 1

Cases of somatoparaphrenia following unilateral parietal lesions

\begin{tabular}{|c|c|c|c|c|c|c|}
\hline $\begin{array}{l}\text { Age at } \\
\text { onset, gender } \\
\text { and hand } \\
\text { preference }\end{array}$ & $\begin{array}{l}\text { Locus of } \\
\text { the lesion }\end{array}$ & Lesion etiology & $\begin{array}{l}\text { Somatic and other } \\
\text { symptoms }\end{array}$ & $\begin{array}{l}\text { Clinical considerations } \\
\text { concerning possible } \\
\text { epilepsy }\end{array}$ & $\begin{array}{l}\text { Psychiatric } \\
\text { comorbidity }\end{array}$ & Reference \\
\hline $\begin{array}{l}72 \\
\text { Female } \\
\mathrm{H} ?\end{array}$ & $\begin{array}{l}\text { Right } \\
\text { temporo- } \\
\text { parietal }\end{array}$ & Infarct & $\begin{array}{l}\text { Somatoparaphrenia } \\
\text { (supernumerary left } \\
\text { limb), left hemineg- } \\
\text { lect }\end{array}$ & $\begin{array}{l}\text { No mention of EEG or } \\
\text { seizures }\end{array}$ & $?$ & $\begin{array}{l}\text { Worthington } \\
\text { et al., } \\
1996 \text { [28] }\end{array}$ \\
\hline $\begin{array}{l}64 \\
\text { Female } \\
\text { LH }\end{array}$ & $\begin{array}{l}\text { Right }> \\
\text { Left } \\
\text { parietal }\end{array}$ & Meningioma & $\begin{array}{l}\text { Somatophrenia } \\
\text { ( } 2 \text { legs on each side, } \\
\text { uninterrupted for two } \\
\text { weeks), slight left vi- } \\
\text { suospatial } \\
\text { hemineglect }\end{array}$ & $\begin{array}{l}\text { A few partial seizures } \\
\text { of the left leg are men- } \\
\text { tioned, but EEG is not } \\
\text { mentioned }\end{array}$ & $\begin{array}{l}\text { Not confused or } \\
\text { disoriented }\end{array}$ & $\begin{array}{l}\text { Vuilleumier } \\
\text { et al., } \\
1997 \text { [34] }\end{array}$ \\
\hline $\begin{array}{l}41 \\
\text { Male } \\
\text { RH }\end{array}$ & $\begin{array}{l}\text { Right } \\
\text { parieto- } \\
\text { temporal }\end{array}$ & Hematoma & $\begin{array}{l}\text { Somatoparaphrenia } \\
\text { (supernumerary feet, } \\
\text { hands and fingers on } \\
\text { the left), left hemine- } \\
\text { glect that disapeared } \\
\text { after } 4 \text { months }\end{array}$ & $\begin{array}{l}\text { No mention of EEG or } \\
\text { seizures }\end{array}$ & $\begin{array}{l}\text { Impulsivity in } \\
\text { keeping with his } \\
\text { previous life- } \\
\text { style, "no evid- } \\
\text { ence of psychotic } \\
\text { paranoid ideas } \\
\text { or depressive } \\
\text { symptoms" }\end{array}$ & $\begin{array}{l}\text { Halligan } \\
\text { et al., } \\
1995 \text { [26] }\end{array}$ \\
\hline $\begin{array}{l}50 \\
\text { Female } \\
\text { RH }\end{array}$ & $\begin{array}{l}\text { Right } \\
\text { parietal }\end{array}$ & $\begin{array}{l}\text { Hematoma after } \\
\text { clipped aneurism } \\
\text { due to a artero- } \\
\text { venous malfor- } \\
\text { mation }\end{array}$ & $\begin{array}{l}\text { Bilateral } \\
\text { somatoparaphrenia } \\
\text { (macropsia of self } \\
\text { and others, misiden- } \\
\text { tification of limb), } \\
\text { alien hand syndrome } \\
\text { (LH), no hemineglect }\end{array}$ & $\begin{array}{l}\text { No signs of paroxysm } \\
\text { in EEG, only slowing } \\
\text { over lesion }\end{array}$ & $?$ & $\begin{array}{l}\text { Leiguardia } \\
\text { et al., } \\
1993[35]\end{array}$ \\
\hline $\begin{array}{l}85 \\
\text { Female } \\
\mathrm{H} ?\end{array}$ & $\begin{array}{l}\text { Right } \\
\text { parietal } \\
\text { and } \\
\text { thalamic }\end{array}$ & Infarct & $\begin{array}{l}\text { Somatoparaphrenia } \\
\text { (misidentification } \\
\text { of left body), no } \\
\text { hemineglect }\end{array}$ & $\begin{array}{l}\text { No mention of seizures } \\
\text { or EEG }\end{array}$ & $?$ & $\begin{array}{l}\text { Paulig } \\
\text { et al., } \\
2000 \text { [36] }\end{array}$ \\
\hline $\begin{array}{l}64 \\
\text { Male } \\
\mathrm{H} ?\end{array}$ & $\begin{array}{l}\text { Right } \\
\text { parieto- } \\
\text { temporo- } \\
\text { occipital }\end{array}$ & Infarct & $\begin{array}{l}\text { Somatoparaphrenia } \\
\text { (left arm is a live ba- } \\
\text { by), no hemineglect }\end{array}$ & $\begin{array}{l}\text { No mention of seizures } \\
\text { or EEG }\end{array}$ & $\begin{array}{l}\text { No history of psy- } \\
\text { chiatric } \\
\text { illness, agitation } \\
\text { and delusions fol- } \\
\text { lowing the stroke, } \\
\text { visual and audito- } \\
\text { ry hallucinations }\end{array}$ & $\begin{array}{l}\text { Richardson, } \\
1992 \text { [37] }\end{array}$ \\
\hline $\begin{array}{l}36 \\
\text { Male } \\
\text { RH }\end{array}$ & $\begin{array}{l}\text { Right } \\
\text { parieto- } \\
\text { occipital }\end{array}$ & $\begin{array}{l}\text { Oligodendro- } \\
\text { glioma }\end{array}$ & $\begin{array}{l}\text { Somatoparaphrenia } \\
\text { (autoscopia in left fi- } \\
\text { eld), no hemineglect }\end{array}$ & $\begin{array}{l}\text { EEG revealed only } \\
\text { slowing over the lesion, } \\
\text { no evidence of epilepsy }\end{array}$ & $?$ & $\begin{array}{l}\text { Maillard } \\
\text { et al., } \\
2004[38]\end{array}$ \\
\hline $\begin{array}{l}46 \\
\text { Male } \\
\text { RH }\end{array}$ & $\begin{array}{l}\text { Right } \\
\text { parietal }\end{array}$ & Meningioma & $\begin{array}{l}\text { Bilateral somatopar- } \\
\text { aphrenia (his right } \\
\text { body slides behind } \\
\text { his left, features of } \\
\text { the right face are } \\
\text { "more prominent" } \\
\text { han the left), the } \\
\text { devil controls left } \\
\text { side, auditory and } \\
\text { visual hallucinations, } \\
\text { mild left hemineglect }\end{array}$ & $\begin{array}{l}\text { The patient had epil- } \\
\text { epsy but the soma- } \\
\text { toparaphrenia was con- } \\
\text { tinuous and the interic- } \\
\text { tal EEG was normal }\end{array}$ & $\begin{array}{l}\text { Heavy alcohol } \\
\text { use, depression, } \\
\text { psychosis } \\
\text { and delusions }\end{array}$ & $\begin{array}{l}\text { Nightingale } \\
\text { et al., } \\
1982 \text { [39] }\end{array}$ \\
\hline
\end{tabular}


Table 1, continued

\begin{tabular}{|c|c|c|c|c|c|c|}
\hline $\begin{array}{l}\text { Age at } \\
\text { onset, gender } \\
\text { and hand } \\
\text { preference }\end{array}$ & $\begin{array}{l}\text { Locus of } \\
\text { the lesion }\end{array}$ & Lesion etiology & $\begin{array}{l}\text { Somatic and other } \\
\text { symptoms }\end{array}$ & $\begin{array}{l}\text { Clinical considerations } \\
\text { concerning possible } \\
\text { epilepsy }\end{array}$ & $\begin{array}{l}\text { Psychiatric } \\
\text { comorbidity }\end{array}$ & Reference \\
\hline $\begin{array}{l}69 \\
\text { Female } \\
\mathrm{H} ?\end{array}$ & $\begin{array}{l}\text { Right } \\
\text { parieto- } \\
\text { temporo- } \\
\text { occipital }\end{array}$ & Infarct & $\begin{array}{l}\text { Somatoparaphrenia } \\
\text { (misidentification of } \\
\text { sentient left arm and } \\
\text { thumb), anosagnosia, } \\
\text { left hemineglect }\end{array}$ & $\begin{array}{l}\text { No mention of EEG or } \\
\text { seizures }\end{array}$ & Logorhea & $\begin{array}{l}\text { Rode } \\
\text { et al., } \\
1992 \text { [27] }\end{array}$ \\
\hline $\begin{array}{l}63 \\
\text { Male } \\
\text { RH }\end{array}$ & $\begin{array}{l}\text { Right } \\
\text { parieto- } \\
\text { fronto- } \\
\text { temporal }\end{array}$ & Infarct & $\begin{array}{l}\text { Bilateral } \\
\text { somatoparaphrenia } \\
\text { (midline limb), } \\
\text { apraxia, spatial } \\
\text { agraphia, acalculia, } \\
\text { left hemineglect }\end{array}$ & $\begin{array}{l}\text { Right fronto-temporal } \\
\text { slowing of EEG }\end{array}$ & $\begin{array}{l}\text { Psychotic } \\
\text { episode, } \\
\text { delusions, } \\
\text { mania, psychosis, } \\
\text { confabulation, } \\
\text { depression and } \\
\text { paranoia }\end{array}$ & $\begin{array}{l}\text { Berthier } \\
\text { et al., } \\
1987 \text { [40] }\end{array}$ \\
\hline $\begin{array}{l}86 \\
\text { Female } \\
\text { RH }\end{array}$ & $\begin{array}{l}\text { Right } \\
\text { parieto- } \\
\text { fronto- } \\
\text { temporal }\end{array}$ & Emboly & $\begin{array}{l}\text { Somatoparaphrenia } \\
\text { (misidentification } \\
\text { of sentient left hand), } \\
\text { anosagnosia, left } \\
\text { hemineglect }\end{array}$ & $\begin{array}{l}\text { No mention of EEG or } \\
\text { seizures }\end{array}$ & $\begin{array}{l}\text { No history of psy- } \\
\text { chiatric illness }\end{array}$ & $\begin{array}{l}\text { Assal, } \\
1983[41]\end{array}$ \\
\hline $\begin{array}{l}84 \\
\text { Female } \\
\text { RH }\end{array}$ & $\begin{array}{l}\text { Right } \\
\text { fronto- } \\
\text { parieto- } \\
\text { occipital }\end{array}$ & Infarct & $\begin{array}{l}\text { Somatoparaphrenia } \\
\text { (attributes sentient } \\
\text { left limb to some- } \\
\text { one else), visual left } \\
\text { hemineglect }\end{array}$ & $\begin{array}{l}\text { No EEG or seizures } \\
\text { reported }\end{array}$ & $?$ & $\begin{array}{l}\text { Bisiach } \\
\text { et al., } \\
1991 \text { [42] }\end{array}$ \\
\hline $\begin{array}{l}77 \\
\text { Female } \\
\text { RH }\end{array}$ & $\begin{array}{l}\text { Right } \\
\text { fronto- } \\
\text { parieto- } \\
\text { temporal } \\
\text { + putamen } \\
\text { and } \\
\text { pallidum }\end{array}$ & Hemorrhage & $\begin{array}{l}\text { Somatoparaphrenia } \\
\text { (her left sensient } \\
\text { hand belongs to } \\
\text { someone else), left } \\
\text { hemineglect, } \\
\text { anosognosia }\end{array}$ & $\begin{array}{l}\text { No EEG or seizures } \\
\text { reported }\end{array}$ & $\begin{array}{l}\text { "Oriented in time } \\
\text { and space and did } \\
\text { not show any oth- } \\
\text { er sign of mental } \\
\text { deterioration" }\end{array}$ & $\begin{array}{l}\text { Bottini } \\
\text { et al., } \\
2002 \text { [12] }\end{array}$ \\
\hline $\begin{array}{l}85 \\
\text { Female } \\
\mathrm{H} ?\end{array}$ & $\begin{array}{l}\text { Right pari- } \\
\text { etal and } \\
\text { thalamic }\end{array}$ & Infarct & $\begin{array}{l}\text { Somatoparaphrenia } \\
\text { (misidentification } \\
\text { of left body), left } \\
\text { hemineglect }\end{array}$ & $\begin{array}{l}\text { No mention of seizures } \\
\text { or EEG }\end{array}$ & $?$ & $\begin{array}{l}\text { Paulig } \\
\text { et al., } \\
2000 \text { [36] }\end{array}$ \\
\hline $\begin{array}{l}76 \\
\text { Female } \\
\mathrm{H} ?\end{array}$ & $\begin{array}{l}\text { Right } \\
\text { parieto- } \\
\text { occipital }\end{array}$ & Infarct & $\begin{array}{l}\text { Somatoparaphrenia } \\
\text { (content unspecifi- } \\
\text { ed), left hemiplegia, } \\
\text { left neglect }\end{array}$ & $\begin{array}{l}\text { No mention of EEG or } \\
\text { seizures }\end{array}$ & $?$ & $\begin{array}{l}\text { Ramachan- } \\
\text { dran, } \\
1996 \text { [43] }\end{array}$ \\
\hline $\begin{array}{l}57 \\
\text { Female } \\
\mathrm{H} ?\end{array}$ & $\begin{array}{l}\text { Right } \\
\text { hemisph- } \\
\text { ere }\end{array}$ & $\begin{array}{l}\text { Several hemorr- } \\
\text { hages }\end{array}$ & $\begin{array}{l}\text { Somatoparaphrenia } \\
\text { (supernumerary } \\
\text { hands), no hemineg- } \\
\text { lect }\end{array}$ & $\begin{array}{l}\text { EEG slowing over the } \\
\text { right hemisphere, no } \\
\text { mention of seizures }\end{array}$ & Depression & $\begin{array}{l}\text { Weinstein } \\
\text { et al., } \\
1954 \text { [44] }\end{array}$ \\
\hline $\begin{array}{l}77 \\
\text { Male } \\
\text { RH }\end{array}$ & $\begin{array}{l}\text { Right } \\
\text { occipito- } \\
\text { parieto- } \\
\text { temporal }\end{array}$ & Infarct & $\begin{array}{l}\text { Somatoparaphrenia } \\
\text { (supernumerary } \\
\text { arms) on the right } \\
\text { hemibody, left } \\
\text { hemineglect }\end{array}$ & $\begin{array}{l}\text { No mention of EEG or } \\
\text { seizures }\end{array}$ & $?$ & $\begin{array}{l}\text { Sellal, } \\
1996[45]\end{array}$ \\
\hline $\begin{array}{l}70 \\
\text { Female } \\
\text { H? }\end{array}$ & $\begin{array}{l}\text { Right } \\
\text { parieto- } \\
\text { temporal }\end{array}$ & $\begin{array}{l}\text { Atrophy } \\
\text { (ganglionic } \\
\text { degeneration) }\end{array}$ & $\begin{array}{l}\text { Somatoparaphrenia } \\
\text { (misidentification of } \\
\text { the left hand), no } \\
\text { hemineglect }\end{array}$ & $\begin{array}{l}\text { EEG slowing over the } \\
\text { lesion, right hypoper- } \\
\text { fusion on SPECT, no } \\
\text { mention of seizures }\end{array}$ & $?$ & $\begin{array}{l}\text { Carrilho } \\
\text { et al., } \\
2001 \text { [46] }\end{array}$ \\
\hline
\end{tabular}


Table 1, continued

\begin{tabular}{|c|c|c|c|c|c|c|}
\hline $\begin{array}{l}\text { Age at } \\
\text { onset, gender } \\
\text { and hand } \\
\text { preference }\end{array}$ & $\begin{array}{l}\text { Locus of } \\
\text { the lesion }\end{array}$ & Lesion etiology & $\begin{array}{l}\text { Somatic and other } \\
\text { symptoms }\end{array}$ & $\begin{array}{l}\text { Clinical considerations } \\
\text { concerning possible } \\
\text { epilepsy }\end{array}$ & $\begin{array}{l}\text { Psychiatric } \\
\text { comorbidity }\end{array}$ & Reference \\
\hline $\begin{array}{l}75 \\
\text { Female } \\
\text { H? }\end{array}$ & $\begin{array}{l}\text { Right } \\
\text { parieto- } \\
\text { temporal }\end{array}$ & Atrophy & $\begin{array}{l}\text { Somatoparaphrenia } \\
\text { (misidentification of } \\
\text { the left hand), no } \\
\text { hemineglect }\end{array}$ & $\begin{array}{l}\text { No mention of EEG or } \\
\text { seizures }\end{array}$ & $?$ & $\begin{array}{l}\text { Carrilho } \\
\text { et al., } \\
2001 \text { [46] }\end{array}$ \\
\hline $\begin{array}{l}63 \\
\text { Male } \\
\mathrm{H} ?\end{array}$ & $\begin{array}{l}\text { Left }> \\
\text { Right } \\
\text { parietal }\end{array}$ & Atrophy & $\begin{array}{l}\text { Somatoparaphrenia } \\
\text { (misidentification of } \\
\text { the right hand), right } \\
\text { hemineglect }\end{array}$ & $\begin{array}{l}\text { EEG slowing over the } \\
\text { lesion, left }>\text { right } \\
\text { temporo-parietal } \\
\text { hypoperfusion } \\
\text { on SPECT, no mention } \\
\text { of seizures }\end{array}$ & $?$ & $\begin{array}{l}\text { Carrilho } \\
\text { et al., } \\
2001 \text { [46] }\end{array}$ \\
\hline $\begin{array}{l}60 \\
\text { Female } \\
\text { H? }\end{array}$ & $\begin{array}{l}\text { Right } \\
\text { parietal }\end{array}$ & Infarct & $\begin{array}{l}\text { Somatoparaphrenia } \\
\text { (misidentification of } \\
\text { the left hand), no } \\
\text { hemineglect }\end{array}$ & $\begin{array}{l}\text { No mention of EEG or } \\
\text { seizures }\end{array}$ & $?$ & $\begin{array}{l}\text { Carrilho } \\
\text { et al., } \\
2001 \text { [46] }\end{array}$ \\
\hline $\begin{array}{l}35 \\
\text { Male } \\
\mathrm{H} ?\end{array}$ & $\begin{array}{l}\text { Right } \\
\text { capsulo- } \\
\text { lenticular, } \\
\text { parieto- } \\
\text { frontal }\end{array}$ & Hematoma & $\begin{array}{l}\text { Somatoparaphrenia } \\
\text { (supernumerary left } \\
\text { arm or leg), left } \\
\text { hemineglect }\end{array}$ & $\begin{array}{l}\text { No mention of EEG or } \\
\text { seizures }\end{array}$ & $?$ & $\begin{array}{l}\text { Donnet } \\
\text { et al., } \\
1997 \text { [25] }\end{array}$ \\
\hline $\begin{array}{l}61 \\
\text { Female } \\
\text { H? }\end{array}$ & $\begin{array}{l}\text { Right } \\
\text { sub- } \\
\text { parietal } \\
\text { white } \\
\text { matter }\end{array}$ & Hemorrhage & $\begin{array}{l}\text { Somatoparaphrenia } \\
\text { (supernumerary left } \\
\text { arm), no hemineglect }\end{array}$ & $\begin{array}{l}\text { No mention of EEG or } \\
\text { seizures }\end{array}$ & $\begin{array}{l}\text { No delusions or } \\
\text { confabulation, re- } \\
\text { active depression }\end{array}$ & $\begin{array}{l}\text { Canavero } \\
\text { et al., } \\
1999[47]\end{array}$ \\
\hline $\begin{array}{l}36 \\
\text { Male } \\
\mathrm{H} ?\end{array}$ & $\begin{array}{l}\text { Right } \\
\text { parietal }\end{array}$ & Aneurysm & $\begin{array}{l}\text { Somatoparaphrenia } \\
\text { (misidentification of } \\
\text { the left hemibody, su- } \\
\text { pernumerary legs and } \\
\text { arms), no hemineg- } \\
\text { lect }\end{array}$ & $\begin{array}{l}\text { No mention of EEG or } \\
\text { seizures }\end{array}$ & $?$ & $\begin{array}{l}\text { Fredericks, } \\
1963 \text { [48] }\end{array}$ \\
\hline $\begin{array}{l}26 \\
\text { Male } \\
\mathrm{H} ?\end{array}$ & $\begin{array}{l}\text { Right } \\
\text { parietal }\end{array}$ & $\begin{array}{l}\text { Penetrating } \\
\text { wound }\end{array}$ & $\begin{array}{l}\text { Somatoparaphrenia } \\
\text { (autoscopia, sensa- } \\
\text { tion that his body was } \\
\text { dislocated, macrosc- } \\
\text { opia), no hemineglect }\end{array}$ & $\begin{array}{l}\text { No mention of EEG or } \\
\text { seizures }\end{array}$ & $\begin{array}{l}\text { No previous ex- } \\
\text { perience of deper- } \\
\text { sonnalization or } \\
\text { any other psychi- } \\
\text { atric disturbance }\end{array}$ & $\begin{array}{l}\text { Lunn, } \\
1970 \text { [49] }\end{array}$ \\
\hline
\end{tabular}

Note. None of these cases were reported to have aphasic symptoms.

spheric specialization. Presence of aphasic symptoms was systematically noted. See Table 2.

All four symptoms of Gerstmann's syndrome (agraphia, acalculia, right-left confusion, finger agnosia) were thought by Gerstmann [16] to be high-order disorders of "body image". The rationale for acalculia being a disorder of body schema is that we learn to count with our fingers. The rationale for agraphia being a disorder of body schema is that the agraphia is postulated to be peripheral rather than central, i.e., putatively proprioceptive and/or apraxic. It is frequently argued that Gerstmann's syndrome always comprises and is essentially explainable as a "defective process of mental manipulation of images" [52-55]. Several authors have believed that Gerstmann's syndrome is a form of aphasia [56-58] and one group has even specified that the aphasia is semantic and that the main problem is a breakdown of the part/whole dialectic [59]. Presence of aphasia weakens our test of the psychic tonus model because aphasia falls under the orbit of a form of hemispheric specialization (linguistic) which we fear may be orthogonal to the issue at hand (though we are not sure of that yet). We therefore documented any mention of aphasia. Others have argued that the syndrome does not exist in pure form. However, all the controversy does not detract from the fact that bilateral finger agnosia and right-left confusion (especially relative to self) are obvious defects of body schema of 
Table 2

Cases of autotopagnosia or Gerstmann's syndrome following a unilateral parietal lesion

\begin{tabular}{|c|c|c|c|c|c|c|}
\hline $\begin{array}{l}\text { Age at } \\
\text { onset, gender } \\
\text { and hand } \\
\text { preference }\end{array}$ & $\begin{array}{l}\text { Lesion } \\
\text { locus }\end{array}$ & Etiology & $\begin{array}{l}\text { Somatic and } \\
\text { other symptoms }\end{array}$ & $\begin{array}{l}\text { Clinical considerations } \\
\text { concerning } \\
\text { possible epilepsy }\end{array}$ & $\begin{array}{l}\text { Psychiatric } \\
\text { comorbidity/ } \\
\text { aphasia }\end{array}$ & Reference \\
\hline $\begin{array}{l}49 \\
\text { Male } \\
\text { H? }\end{array}$ & $\begin{array}{l}\text { Left } \\
\text { parietal }\end{array}$ & Glioma & $\begin{array}{l}\text { Autotopagnosia } \\
\text { (bilateral), no aphasia }\end{array}$ & $\begin{array}{l}\text { Diffuse slowing of } \\
\text { EEG }\end{array}$ & $\begin{array}{l}\text { No mention of } \\
\text { psychiatric } \\
\text { comorbidity, } \\
\text { no aphasia }\end{array}$ & $\begin{array}{l}\text { De Renzi \& } \\
\text { Faglioni, } \\
1963 \text { [60] }\end{array}$ \\
\hline $\begin{array}{l}64 \\
\text { Male } \\
\text { RH }\end{array}$ & $\begin{array}{l}\text { Left } \\
\text { parietal }\end{array}$ & Tumor & $\begin{array}{l}\text { Autotopagnosia } \\
\text { (bilateral), no aphasia }\end{array}$ & $\begin{array}{l}\text { No mention of } \\
\text { EEG or seizures }\end{array}$ & $\begin{array}{l}\text { No mention of } \\
\text { psychiatric } \\
\text { comorbidity, } \\
\text { no aphasia }\end{array}$ & $\begin{array}{l}\text { De Renzi \& } \\
\text { Scotti, } \\
1970[61]\end{array}$ \\
\hline $\begin{array}{l}59 \\
\text { Male } \\
\text { RH }\end{array}$ & $\begin{array}{l}\text { Left } \\
\text { parietal }\end{array}$ & $\begin{array}{l}\text { Cystic tumor } \\
\text { (metastatic } \\
\text { carcinoma) }\end{array}$ & $\begin{array}{l}\text { Autotopagnosia (bi- } \\
\text { lateral), Gerstmann } \\
\text { syndrome, ideomotor } \\
\text { apraxia, no aphasia }\end{array}$ & $\begin{array}{l}\text { No mention of } \\
\text { EEG or seizures }\end{array}$ & $\begin{array}{l}\text { No mention of } \\
\text { psychiatric } \\
\text { comorbidity, } \\
\text { no aphasia }\end{array}$ & $\begin{array}{l}\text { Ogden, } \\
1985[62]\end{array}$ \\
\hline $\begin{array}{l}67 \\
\text { Male } \\
\text { RH }\end{array}$ & $\begin{array}{l}\text { Left } \\
\text { parietal }\end{array}$ & $\begin{array}{l}\text { Encephaloma- } \\
\text { lacia }\end{array}$ & $\begin{array}{l}\text { Autotopagnosia } \\
\text { (bilateral), dyscalcu- } \\
\text { lia, dysgraphia, no } \\
\text { aphasia }\end{array}$ & $\begin{array}{l}\text { No mention of } \\
\text { EEG or seizures }\end{array}$ & $\begin{array}{l}\text { No mention of } \\
\text { psychiatric } \\
\text { comorbidity, } \\
\text { aphasia }\end{array}$ & $\begin{array}{l}\text { Denes } \\
\text { et al., } \\
2000[13]\end{array}$ \\
\hline $\begin{array}{l}74 \\
\text { Female } \\
\text { LH }\end{array}$ & $\begin{array}{l}\text { Right } \\
\text { parieto- } \\
\text { temporo- } \\
\text { occipital }\end{array}$ & $\begin{array}{l}\text { Hemorrhagic } \\
\text { infarct }\end{array}$ & $\begin{array}{l}\text { Autotopagnosia (bi- } \\
\text { lateral), limb apraxia, } \\
\text { neglect syndrome, } \\
\text { no aphasia }\end{array}$ & $\begin{array}{l}\text { No mention of } \\
\text { EEG or seizures }\end{array}$ & $\begin{array}{l}\text { No mention of } \\
\text { psychiatric } \\
\text { comorbidity, } \\
\text { no aphasia }\end{array}$ & $\begin{array}{l}\text { Verstichel } \\
\text { et al., } \\
1994 \text { [63] }\end{array}$ \\
\hline $\begin{array}{l}64 \\
\text { Female } \\
\text { H? }\end{array}$ & $\begin{array}{l}\text { Left } \\
\text { parietal }\end{array}$ & $\begin{array}{l}\text { Anaplastic } \\
\text { astrocytoma }\end{array}$ & $\begin{array}{l}\text { Autotopagnosia (bi- } \\
\text { lateral), anomia }\end{array}$ & $\begin{array}{l}\text { No mention of } \\
\text { EEG or seizures }\end{array}$ & $\begin{array}{l}\text { No mention of } \\
\text { psychiatric } \\
\text { comorbidity, } \\
\text { anomia }\end{array}$ & $\begin{array}{l}\text { Baldini } \\
\text { et al., } \\
1978[5]\end{array}$ \\
\hline $\begin{array}{l}56 \\
\text { Male } \\
\text { RH }\end{array}$ & $\begin{array}{l}\text { Left } \\
\text { parieto- } \\
\text { occipital }\end{array}$ & Hemorrhage & $\begin{array}{l}\text { Autotopagnosia } \\
\text { (bilateral), } \\
\text { mild dysphasia }\end{array}$ & $\begin{array}{l}\text { Left EEG slowing } \\
\text { without epileptiform } \\
\text { signs }\end{array}$ & $\begin{array}{l}\text { no mention of } \\
\text { psychiatric } \\
\text { comorbidity, mild } \\
\text { dysphasia }\end{array}$ & $\begin{array}{l}\text { Poncet } \\
\text { et al., } \\
1971 \text { [59] }\end{array}$ \\
\hline $\begin{array}{l}71 \\
\text { Female } \\
\text { LH }\end{array}$ & $\begin{array}{l}\text { Right pari- } \\
\text { etal (right } \\
\text { hemisphere } \\
\text { dominant } \\
\text { for } \\
\text { language) }\end{array}$ & $\begin{array}{l}\text { Encephalomal- } \\
\text { acia }\end{array}$ & $\begin{array}{l}\text { Autotopagnosia (bi- } \\
\text { lateral), aphasia }\end{array}$ & $\begin{array}{l}\text { No mention of } \\
\text { EEG or seizures }\end{array}$ & $\begin{array}{l}\text { No mention of } \\
\text { psychiatric } \\
\text { comorbidity, } \\
\text { aphasia }\end{array}$ & $\begin{array}{l}\text { Denes } \\
\text { et al., } \\
2000[13]\end{array}$ \\
\hline $\begin{array}{l}77 \\
\text { Female } \\
\text { RH }\end{array}$ & $\begin{array}{l}\text { Left } \\
\text { parieto- } \\
\text { temporo- } \\
\text { occipital }\end{array}$ & Infarct & $\begin{array}{l}\text { Autotopagnosia } \\
\text { (bilateral), ideomotor } \\
\text { apraxia, aphasia }\end{array}$ & $\begin{array}{l}\text { No mention of } \\
\text { EEG or seizures }\end{array}$ & $\begin{array}{l}\text { No mention of } \\
\text { psychiatric } \\
\text { comorbidity, } \\
\text { aphasia }\end{array}$ & $\begin{array}{l}\text { Schwoebel } \\
\text { et al., } \\
2001[14]\end{array}$ \\
\hline $\begin{array}{l}74 \\
\text { Female } \\
\text { RH }\end{array}$ & $\begin{array}{l}\text { Left } \\
\text { parieto- } \\
\text { occipital }\end{array}$ & $\begin{array}{l}\text { Metastasic } \\
\text { melanoma }\end{array}$ & $\begin{array}{l}\text { Autotopagnosia (bi- } \\
\text { lateral), mild aphasia }\end{array}$ & $\begin{array}{l}\text { No mention of } \\
\text { EEG or seizures }\end{array}$ & $\begin{array}{l}\text { No mention of } \\
\text { psychiatric } \\
\text { comorbidity, } \\
\text { mild aphasia }\end{array}$ & $\begin{array}{l}\text { Semenza, } \\
1988[64]\end{array}$ \\
\hline $\begin{array}{l}71 \\
\text { Male } \\
\text { H? }\end{array}$ & $\begin{array}{l}\text { Left } \\
\text { parieto- } \\
\text { occipital }\end{array}$ & Tissue softening & $\begin{array}{l}\text { Complete autotopag- } \\
\text { nosia with conseq- } \\
\text { uent Gerstmann' } \\
\text { syndrome }\end{array}$ & $\begin{array}{l}\text { Normal neurological } \\
\text { exam, no mention of } \\
\text { imagery }\end{array}$ & $\begin{array}{l}\text { No mention of } \\
\text { psychiatric } \\
\text { comorbidity, } \\
\text { no aphasia }\end{array}$ & $\begin{array}{l}\text { Nielsen, } \\
1946[65]\end{array}$ \\
\hline $\begin{array}{l}59 \\
\text { Male } \\
\text { RH }\end{array}$ & $\begin{array}{l}\text { Left } \\
\text { parietal }\end{array}$ & Stroke & $\begin{array}{l}\text { Gerstmann's } \\
\text { syndrome, (bilateral } \\
\text { finger agnosia docu- } \\
\text { mented), no aphasia }\end{array}$ & $\begin{array}{l}\text { No mention of } \\
\text { EEG or seizures }\end{array}$ & $\begin{array}{l}\text { No mention of } \\
\text { psychiatric } \\
\text { comorbidity, } \\
\text { mild word finding } \\
\text { difficulties }\end{array}$ & $\begin{array}{l}\text { Varney, } \\
1984[66]\end{array}$ \\
\hline
\end{tabular}


Table 2, continued

\begin{tabular}{|c|c|c|c|c|c|c|}
\hline $\begin{array}{l}\text { Age at } \\
\text { onset, gender } \\
\text { and hand } \\
\text { preference }\end{array}$ & $\begin{array}{l}\text { Locus of } \\
\text { the lesion }\end{array}$ & Lesion etiology & $\begin{array}{l}\text { Somatic and other } \\
\text { symptoms }\end{array}$ & $\begin{array}{l}\text { Clinical considerations } \\
\text { concerning possible } \\
\text { epilepsy }\end{array}$ & $\begin{array}{l}\text { Psychiatric } \\
\text { comorbidity }\end{array}$ & Reference \\
\hline $\begin{array}{l}48 \\
\text { Female } \\
\text { H? }\end{array}$ & $\begin{array}{l}\text { Left } \\
\text { parieto- } \\
\text { occipital }\end{array}$ & $\begin{array}{l}\text { Tumor } \\
\text { (multiform } \\
\text { spongioblastoma) }\end{array}$ & $\begin{array}{l}\text { Gerstmann's syn- } \\
\text { drome (bilateral fin- } \\
\text { ger agnosia docu- } \\
\text { mented), no aphasia }\end{array}$ & $\begin{array}{l}\text { No mention of } \\
\text { EEG, seizures or psy- } \\
\text { chiatric comorbidity }\end{array}$ & $\begin{array}{l}\text { No mention of } \\
\text { psychiatric } \\
\text { comorbidity, } \\
\text { no aphasia }\end{array}$ & $\begin{array}{l}\text { Arbuse, } \\
1947 \text { [67] }\end{array}$ \\
\hline $\begin{array}{l}60 \\
\text { Female } \\
\text { H? }\end{array}$ & $\begin{array}{l}\text { Left } \\
\text { parieto- } \\
\text { occipital }\end{array}$ & Infarct & $\begin{array}{l}\text { Gerstmann's syn- } \\
\text { drome (bilateral fin- } \\
\text { ger agnosia docu- } \\
\text { mented), no aphasia }\end{array}$ & $\begin{array}{l}\text { No paroxysms in } \\
\text { EEG, slowing over } \\
\text { lesion }\end{array}$ & $\begin{array}{l}\text { No mention of } \\
\text { psychiatric } \\
\text { comorbidity, } \\
\text { no aphasia }\end{array}$ & $\begin{array}{l}\text { Perez- } \\
\text { Blanco } \\
\text { et al., } \\
1989 \text { [68] }\end{array}$ \\
\hline $\begin{array}{l}64 \\
\text { Male } \\
\text { RH }\end{array}$ & $\begin{array}{l}\text { Right } \\
\text { parietal }\end{array}$ & Infarct & $\begin{array}{l}\text { Gerstmann's } \\
\text { syndrome, (bilateral } \\
\text { finger agnosia docu- } \\
\text { mented), no aphasia }\end{array}$ & $\begin{array}{l}\text { No mention of } \\
\text { EEG or seizures }\end{array}$ & $\begin{array}{l}\text { No mention of } \\
\text { psychiatric } \\
\text { comorbidity, } \\
\text { no aphasia }\end{array}$ & $\begin{array}{l}\text { Calvo- } \\
\text { Romero, } \\
2000[69]\end{array}$ \\
\hline $\begin{array}{l}71 \\
\text { Female } \\
\text { RH }\end{array}$ & $\begin{array}{l}\text { Left } \\
\text { fronto- } \\
\text { parietal }\end{array}$ & Hematoma & $\begin{array}{l}\text { Gerstmann's } \\
\text { syndrome, (bilateral } \\
\text { finger agnosia docu- } \\
\text { mented), no aphasia }\end{array}$ & $\begin{array}{l}\text { No mention of } \\
\text { EEG or seizures }\end{array}$ & $\begin{array}{l}\text { No mention of } \\
\text { psychiatric } \\
\text { comorbidity, mild } \\
\text { word finding im- } \\
\text { pairment, } \\
\text { paraphasic errors }\end{array}$ & $\begin{array}{l}\text { Maeshima } \\
\text { et al., } \\
1998[70]\end{array}$ \\
\hline $\begin{array}{l}85 \\
\text { Male } \\
\text { ambidextr- } \\
\text { ous }\end{array}$ & $\begin{array}{l}\text { Left } \\
\text { parietal }\end{array}$ & Ischemic stroke & $\begin{array}{l}\text { Gerstmann's syn- } \\
\text { drome (bilateral fin- } \\
\text { ger agnosia docu- } \\
\text { mented), no aphasia }\end{array}$ & $\begin{array}{l}\text { No mention of } \\
\text { EEG or seizures }\end{array}$ & $\begin{array}{l}\text { No mention of } \\
\text { psychiatric } \\
\text { comorbidity, } \\
\text { no aphasia }\end{array}$ & $\begin{array}{l}\text { Carota } \\
\text { et al., } \\
2004[52]\end{array}$ \\
\hline $\begin{array}{l}57 \\
\text { Female } \\
\text { LH }\end{array}$ & $\begin{array}{l}\text { Right } \\
\text { parietal }\end{array}$ & Stroke & $\begin{array}{l}\text { Gerstmann's } \\
\text { syndrome, (bilateral } \\
\text { finger agnosia docu- } \\
\text { mented), dysphasia }\end{array}$ & $\begin{array}{l}\text { No mention of } \\
\text { EEG or seizures }\end{array}$ & $\begin{array}{l}\text { No mention of } \\
\text { psychiatric } \\
\text { comorbidity, } \\
\text { dysphasia }\end{array}$ & $\begin{array}{l}\text { Moore } \\
\text { et al., } \\
1991[71]\end{array}$ \\
\hline $\begin{array}{l}69 \\
\text { Male } \\
\text { ambidextr- } \\
\text { ous }\end{array}$ & $\begin{array}{l}\text { Left } \\
\text { parieto- } \\
\text { occipital }\end{array}$ & Hemorrhage & $\begin{array}{l}\text { Gerstmann's syn- } \\
\text { drome (bilateral fin- } \\
\text { ger agnosia docu- } \\
\text { mented), no aphasia }\end{array}$ & $\begin{array}{l}\text { No mention of } \\
\text { EEG or seizures }\end{array}$ & $\begin{array}{l}\text { No mention of } \\
\text { psychiatric } \\
\text { comorbidity, } \\
\text { no aphasia }\end{array}$ & $\begin{array}{l}\text { Dozono } \\
\text { et al., } \\
1997[72]\end{array}$ \\
\hline $\begin{array}{l}52 \\
\text { Male } \\
\text { RH }\end{array}$ & $\begin{array}{l}\text { Left } \\
\text { parietal }\end{array}$ & Stroke & $\begin{array}{l}\text { Gerstmann's syn- } \\
\text { drome (bilateral fin- } \\
\text { ger agnosia docu- } \\
\text { mented), no aphasia }\end{array}$ & $\begin{array}{l}\text { No EEG or seizures } \\
\text { reported }\end{array}$ & $\begin{array}{l}\text { Euphoria, } \\
\text { no aphasia }\end{array}$ & $\begin{array}{l}\text { Sobota } \\
\text { et al., } \\
1985[73]\end{array}$ \\
\hline $\begin{array}{l}44 \\
\text { Male } \\
\text { RH }\end{array}$ & $\begin{array}{l}\text { Left } \\
\text { parietal }\end{array}$ & $\begin{array}{l}\text { Penetrating head } \\
\text { injury }\end{array}$ & $\begin{array}{l}\text { Gerstmann's syn- } \\
\text { drome (bilateral fin- } \\
\text { ger agnosia docu- } \\
\text { mented), no aphasia }\end{array}$ & $\begin{array}{l}\text { No mention of } \\
\text { EEG or seizures }\end{array}$ & $\begin{array}{l}\text { No mention of } \\
\text { psychiatric } \\
\text { comorbidity, } \\
\text { no aphasia }\end{array}$ & $\begin{array}{l}\text { Mazzoni } \\
\text { et al., } \\
1990[74]\end{array}$ \\
\hline $\begin{array}{l}59 \\
\text { Male } \\
\text { RH }\end{array}$ & $\begin{array}{l}\text { Left } \\
\text { parietal }\end{array}$ & Infarct & $\begin{array}{l}\text { Gerstmann's syn- } \\
\text { drome (bilateral fin- } \\
\text { ger agnosia docu- } \\
\text { mented), impairment } \\
\text { of visual rotation, no } \\
\text { apraxia or aphasia }\end{array}$ & $\begin{array}{l}\text { No mention of } \\
\text { EEG or seizures }\end{array}$ & $\begin{array}{l}\text { No mention of } \\
\text { psychiatric } \\
\text { comorbidity, } \\
\text { no aphasia }\end{array}$ & $\begin{array}{l}\text { Mayer } \\
\text { et al., } \\
1999[75]\end{array}$ \\
\hline $\begin{array}{l}72 \\
\text { Female } \\
\text { RH }\end{array}$ & $\begin{array}{l}\text { Left } \\
\text { parietal }\end{array}$ & $\begin{array}{l}\text { Glioblastoma } \\
\text { multiforme }\end{array}$ & $\begin{array}{l}\text { Gerstmann's syn- } \\
\text { drome with bilateral } \\
\text { toe agnosia (bilateral } \\
\text { finger agnosia docu- } \\
\text { mented), no aphasia }\end{array}$ & $\begin{array}{l}\text { No mention of } \\
\text { seizures or EEG }\end{array}$ & $\begin{array}{l}\text { No history of } \\
\text { psychiatric } \\
\text { illness, } \\
\text { no aphasia }\end{array}$ & $\begin{array}{l}\text { Tucha } \\
\text { et al., } \\
1997[76]\end{array}$ \\
\hline
\end{tabular}


Table 2, continued

\begin{tabular}{|c|c|c|c|c|c|c|}
\hline $\begin{array}{l}\text { Age at } \\
\text { onset, gender } \\
\text { and hand } \\
\text { preference }\end{array}$ & $\begin{array}{l}\text { Locus of } \\
\text { the lesion }\end{array}$ & Lesion etiology & $\begin{array}{l}\text { Somatic and other } \\
\text { symptoms }\end{array}$ & $\begin{array}{l}\text { Clinical considerations } \\
\text { concerning possible } \\
\text { epilepsy }\end{array}$ & $\begin{array}{l}\text { Psychiatric } \\
\text { comorbidity }\end{array}$ & Reference \\
\hline $\begin{array}{l}58 \\
\text { Male } \\
\text { RH }\end{array}$ & $\begin{array}{l}\text { Left } \\
\text { parietal }\end{array}$ & Stroke & $\begin{array}{l}\text { Gerstmann's } \\
\text { syndrome, (bilateral } \\
\text { finger agnosia docu- } \\
\text { mented), } \\
\text { semantic dysphasia }\end{array}$ & $\begin{array}{l}\text { No mention of } \\
\text { EEG or seizures }\end{array}$ & $\begin{array}{l}\text { No mention of } \\
\text { psychiatric } \\
\text { comorbidity, } \\
\text { semantic } \\
\text { dysphasia }\end{array}$ & $\begin{array}{l}\text { Ardila } \\
\text { et al., } \\
2000[56]\end{array}$ \\
\hline $\begin{array}{l}7 \\
\text { Male } \\
\text { H? }\end{array}$ & $\begin{array}{l}\text { Left } \\
\text { parietal }\end{array}$ & Infarct & $\begin{array}{l}\text { Gerstmann's } \\
\text { syndrome, no aphasia }\end{array}$ & $\begin{array}{l}\text { No mention of } \\
\text { EEG or seizures }\end{array}$ & $\begin{array}{l}\text { No mention of } \\
\text { psychiatric } \\
\text { comorbidity, } \\
\text { no aphasia }\end{array}$ & $\begin{array}{l}\text { Ohtagaki } \\
\text { et al., } \\
1998 \text { [77] }\end{array}$ \\
\hline $\begin{array}{l}12 \\
\text { Male } \\
\mathrm{H} ?\end{array}$ & $\begin{array}{l}\text { Left } \\
\text { parietal }\end{array}$ & Atrophy & $\begin{array}{l}\text { Gerstmann's } \\
\text { syndrome, no aphasia }\end{array}$ & $\begin{array}{l}\text { No mention of } \\
\text { EEG or seizures }\end{array}$ & $\begin{array}{l}\text { No mention of } \\
\text { psychiatric } \\
\text { comorbidity, } \\
\text { no aphasia }\end{array}$ & $\begin{array}{l}\text { Garty } \\
\text { et al., } \\
1989[78]\end{array}$ \\
\hline $\begin{array}{l}64 \\
\text { Male } \\
\text { RH }\end{array}$ & $\begin{array}{l}\text { Left } \\
\text { parietal } \\
\text { (angular } \\
\text { and supra- } \\
\text { marginal } \\
\text { gyri) }\end{array}$ & Infarct & $\begin{array}{l}\text { Gerstmann's } \\
\text { syndrome, no aphasia }\end{array}$ & $\begin{array}{l}\text { No mention of } \\
\text { EEG or seizures }\end{array}$ & $\begin{array}{l}\text { No mention of } \\
\text { psychiatric } \\
\text { comorbidity, } \\
\text { no aphasia }\end{array}$ & $\begin{array}{l}\text { Roeltgen } \\
\text { et al., } \\
1983 \text { [79] }\end{array}$ \\
\hline $\begin{array}{l}65 \\
\text { Male } \\
\text { RH }\end{array}$ & $\begin{array}{l}\text { Left } \\
\text { parieto- } \\
\text { occipital }\end{array}$ & Infarct & $\begin{array}{l}\text { Gerstmann's syn- } \\
\text { drome without alexia, } \\
\text { apraxia or aphasia }\end{array}$ & $\begin{array}{l}\text { No mention of } \\
\text { EEG or seizures }\end{array}$ & $\begin{array}{l}\text { No mention of } \\
\text { psychiatric } \\
\text { comorbidity, } \\
\text { word-finding } \\
\text { difficulty }\end{array}$ & $\begin{array}{l}\text { Levine } \\
\text { et al., } \\
1988 \text { [57] }\end{array}$ \\
\hline $\begin{array}{l}56 \\
\text { Male } \\
\text { RH }\end{array}$ & $\begin{array}{l}\text { Left } \\
\text { parieto- } \\
\text { temporal }\end{array}$ & Infarct & $\begin{array}{l}\text { Gerstmann's } \\
\text { syndrome, no aphasia }\end{array}$ & $\begin{array}{l}\text { EEG slowing over le- } \\
\text { sion, no mention of } \\
\text { seizures }\end{array}$ & $\begin{array}{l}\text { No mention of } \\
\text { psychiatric } \\
\text { comorbidity, } \\
\text { no aphasia }\end{array}$ & $\begin{array}{l}\text { Kinsbourne } \\
\text { et al., } \\
1974 \text { [80] }\end{array}$ \\
\hline $\begin{array}{l}47 \\
\text { Male } \\
\text { RH }\end{array}$ & $\begin{array}{l}\text { Left } \\
\text { parietal }\end{array}$ & Astrocytic glioma & $\begin{array}{l}\text { Gerstmann's } \\
\text { syndrome, dysphasia }\end{array}$ & $\begin{array}{l}\text { No mention of } \\
\text { EEG or seizures }\end{array}$ & $\begin{array}{l}\text { No mention of } \\
\text { psychiatric } \\
\text { comorbidity, } \\
\text { dysphasia }\end{array}$ & $\begin{array}{l}\text { Kinsbourne } \\
\text { et al., } \\
1962 \text { [81] }\end{array}$ \\
\hline $\begin{array}{l}65 \\
\text { Male } \\
\text { LH }\end{array}$ & $\begin{array}{l}\text { Left } \\
\text { parieto- } \\
\text { occipital }\end{array}$ & Meningioma & $\begin{array}{l}\text { Gerstmann's } \\
\text { syndrome, no aphasia }\end{array}$ & $\begin{array}{l}\text { EEG slowing over le- } \\
\text { sion, no mention of } \\
\text { seizures }\end{array}$ & $\begin{array}{l}\text { No mention of } \\
\text { psychiatric } \\
\text { comorbidity, } \\
\text { no aphasia }\end{array}$ & $\begin{array}{l}\text { Kinsbourne, } \\
1962[81]\end{array}$ \\
\hline $\begin{array}{l}7 \\
\text { Male } \\
\text { RH }\end{array}$ & $\begin{array}{l}\text { Left } \\
\text { parieto- } \\
\text { occipital } \\
\text { softening }\end{array}$ & Infarct & $\begin{array}{l}\text { Gerstmann's } \\
\text { syndrome, ADHD, } \\
\text { learning disability, no } \\
\text { aphasia }\end{array}$ & $\begin{array}{l}\text { EEG was normal bilat- } \\
\text { erally, no mention of } \\
\text { seizures }\end{array}$ & $\begin{array}{l}\text { No mention of } \\
\text { psychiatric } \\
\text { comorbidity, } \\
\text { no aphasia }\end{array}$ & $\begin{array}{l}\text { Fournier-Del } \\
\text { Castillo } \\
\text { et al., } \\
2000[82]\end{array}$ \\
\hline $\begin{array}{l}67 \\
\text { Male } \\
\text { RH }\end{array}$ & $\begin{array}{l}\text { Left } \\
\text { parietal }\end{array}$ & Stroke & $\begin{array}{l}\text { Gerstmann's } \\
\text { syndrome, aphasia }\end{array}$ & $\begin{array}{l}\text { No mention of } \\
\text { EEG or seizures }\end{array}$ & $\begin{array}{l}\text { No mention of } \\
\text { psychiatric } \\
\text { comorbidity, } \\
\text { aphasia }\end{array}$ & $\begin{array}{l}\text { Sheimo } \\
\text { et al., } \\
1997[58]\end{array}$ \\
\hline $\begin{array}{l}65 \\
\text { Male } \\
\text { RH }\end{array}$ & $\begin{array}{l}\text { Left } \\
\text { parietal }\end{array}$ & Haematoma & $\begin{array}{l}\text { Gerstmann's } \\
\text { syndrome, no aphasia }\end{array}$ & $\begin{array}{l}\text { No mention of } \\
\text { EEG or seizures }\end{array}$ & $\begin{array}{l}\text { No mention of } \\
\text { psychiatric } \\
\text { comorbidity, } \\
\text { no aphasia }\end{array}$ & $\begin{array}{l}\text { Trillet } \\
\text { et al., } \\
1989 \text { [83] }\end{array}$ \\
\hline $\begin{array}{l}52 \\
\text { Male } \\
\text { RH }\end{array}$ & $\begin{array}{l}\text { Left } \\
\text { parietal }\end{array}$ & Haematoma & $\begin{array}{l}\text { Gerstmann's } \\
\text { syndrome, no aphasia }\end{array}$ & $\begin{array}{l}\text { No mention of } \\
\text { EEG or seizures }\end{array}$ & $\begin{array}{l}\text { No mention of } \\
\text { psychiatric } \\
\text { comorbidity, } \\
\text { no aphasia }\end{array}$ & $\begin{array}{l}\text { Trillet } \\
\text { et al., } \\
1989 \text { [83] }\end{array}$ \\
\hline
\end{tabular}


Table 2, continued

\begin{tabular}{|c|c|c|c|c|c|c|}
\hline $\begin{array}{l}\text { Age at } \\
\text { onset, gender } \\
\text { and hand } \\
\text { preference }\end{array}$ & $\begin{array}{l}\text { Locus of } \\
\text { the lesion }\end{array}$ & Lesion etiology & $\begin{array}{l}\text { Somatic and other } \\
\text { symptoms }\end{array}$ & $\begin{array}{l}\text { Clinical considerations } \\
\text { concerning possible } \\
\text { epilepsy }\end{array}$ & $\begin{array}{l}\text { Psychiatric } \\
\text { comorbidity }\end{array}$ & Reference \\
\hline $\begin{array}{l}68 \\
\text { Male } \\
\text { LH }\end{array}$ & $\begin{array}{l}\text { Right } \\
\text { parietal }\end{array}$ & Infarct & $\begin{array}{l}\text { Gerstmann's } \\
\text { syndrome, no aphasia }\end{array}$ & $\begin{array}{l}\text { No mention of } \\
\text { EEG or seizures }\end{array}$ & $\begin{array}{l}\text { No mention of } \\
\text { psychiatric } \\
\text { comorbidity, } \\
\text { no aphasia }\end{array}$ & $\begin{array}{l}\text { Dehaene } \\
\text { et al., } \\
1997 \text { [84] }\end{array}$ \\
\hline $\begin{array}{l}79 \\
\text { Male } \\
\text { RH }\end{array}$ & $\begin{array}{l}\text { Left } \\
\text { parietal }\end{array}$ & Infarct & $\begin{array}{l}\text { Gerstmann's } \\
\text { syndrome, no aphasia }\end{array}$ & $\begin{array}{l}\text { No mention of } \\
\text { EEG or seizures }\end{array}$ & $\begin{array}{l}\text { No mention of } \\
\text { psychiatric } \\
\text { comorbidity, } \\
\text { no aphasia }\end{array}$ & $\begin{array}{l}\text { Gold } \\
\text { et al., } \\
1995[53]\end{array}$ \\
\hline
\end{tabular}

the agnosic type (though manipulation of images may be a problem as well, and the problem can be verbally mediated), and these two symptoms affect both sides of the body, making them highly relevant tests of the "psychic tonus" model. At any rate, the controversy over Gerstmann's syndrome has resulted in a large set of case reports, useful for testing the psychic tonus model.

Cases of Gerstmann's syndrome following a unilateral parietal lesion which are better suited to testing the psychic tonus model are those where the finger agnosia is documented as bilateral. In Table 3, we noted whether the cases were documented for bilateral agnosia or not. See Table 2.

Of the 37 cases of Table 2, all with a negative disorder of body schema, 32 had a left hemisphere lesion and 5 had a right hemisphere lesion (binomial: $p<0.0005$, two tailed).

Any issue of a general bias in published case reports for lesions in one or the other hemisphere is eschewed by a test of the prediction of a crossed double dissociation. All the post lesion cases with positive syndromes (somatoparaphrenia) $(N=25)$ were therefore compared to all the post lesion cases with a negative syndrome (autotopagnosia, Gerstmann's syndrome) ( $N=$ 37). The crossed double dissociation between lesion side and type of somesthesic disorder is highly significant $\left(\mathrm{Chi}^{2}=40.77, p<0.0005\right.$, two tailed).

\subsection{Secondary analyses for purposes of artefact control (all cases, $N=62$ )}

The main results reported above appear to be highly significant and thus, to be concordant with the psychic tonus model. However, it is important to determine whether that inference test withstands challenges from eventual contamination from extraneous sampling bias.

The analyses to be presented next comprise tests of alternative explanations of the interaction between somesthesic disorder type and lesion side. We operationalized this interaction as a dichotomous variable which we termed "concordance with the psychic tonus model". A left lesion associated with autotopagnosia $\backslash$ Gerstmann syndrome or a right lesion associated with somatoparaphrenia was rated as 1 , the other eventualities were rated as 2 . In the case of a significant relation between "concordance with the psychic tonus model" and an extraneous variable, a second phase of analysis was to be implemented, subjecting the theorical effect to partial correlation - retrenching the variance of the contaminant. This analytical framework supposes that only dichotomous variables may be processed. Consequently all control variables were to be eventually dichotomized if not naturally limited to two levels.

\subsection{Hand writing preference}

Hand writing preference is an important variable because non-right handers present reversed hemispheric specialization in 10 to $30 \%$ of cases. Biased sampling of hand preference could thus bring a caveat to the main result (concordance with the psychic tonus model by lesion side). In this study, there were 32 right handers of which 31 were concordant with the psychic tonus model and 8 left handers or ambidextrals or which 4 were concordant with the psychic tonus model (the other cases' hand preference was not reported). The relation between these two variables is significant, the nonright handers being less concordant with the psychic tonus model $(50 \%)$ than the right handers $(96.9 \%)$. A partial correlation was therefore computed between lesion side and type of somesthesic disorder, controlling for hand writing preference. The psychic tonus effect remained significant despite statistical removal of the effect of handedness $(r p=0.82, p<0.0005)$. 


\subsection{Other control variables}

Locus of the lesion was analyzed because though parietal lobe lesions were a selection criterion, damage elsewhere was not an exclusion criterion, and thus an extra parietal lesion sampling artefact had to be ruled out. Lesion size was analyzed because larger lesions can be thought to produce loss of mental function more often than smaller lesions, and thus a sampling bias could contribute an artefact. Etiology of the lesion was of interest because some etiologies, like tumor, can sometimes irritate and excite brain tissue and create cognitive symptoms [85]. Head trauma can also introduce noise into the data base because it presents with diffuse bilateral damage that is too subtle to be radiologically localized. Cases with old (ex: congenital) lesions could present far more cognitive compensation, adding noise to the inference tests. Presence of a psychiatric comorbidity was analyzed because somatic hallucination or delusion, though less frequent than auditory or visual hallucination, are not rare in psychotics $[86,87]$. The age of the patient is of interest because a bilateral diffuse stress on the brain is associated with aging. Juvenile cases also present less hemispheric specialization than adults. Gender was of interest because women tend to have less focal cognitive representation in the hemispheres and also less functional lateralization. The date of publication is pertinent because MRI technology used nowadays as a diagnostic method is more precise than CT scan. The presence of an EEG was of concern because it brings more precision to lesion localization and reduces chances of contamination by epileptiform activity. The presence/absence of aphasic symptoms is an important control variable because of the well known hemispheric specialization for linguistic abilities (see [88] for a review). However, none of these variables was significantly related to "concordance with the psychic tonus model".

\subsection{More specific analyses (somatoparaphrenic group only, $N=25$ )}

In the neurological literature, it is well known that positive symptoms often occur in the contralateral or the neglected hemibody [89]. In the current study, 20 cases out of 25 had their target symptoms occuring in the hemibody contralateral to the lesion $\left(\mathrm{Chi}^{2}=7.64\right.$, $p=0.054)$. There are however four cases of right hemisphere lesions, thus concordant with the psychic tonus model, for whom the symptoms occured bilaterally. Power was lacking to measure statistically the
Table 3

Distribution of symptom lateralization

\begin{tabular}{cccc}
\hline \multicolumn{4}{c}{ Hemibody where the target symptom occured } \\
\hline Right & Left & Bilateral & Unspecified \\
\hline 3 & 17 & 4 & 1 \\
\hline
\end{tabular}

psychic tonus model inference with these cases only. See Table 3.

In the current data base, there were 12 non neglecting cases and 13 cases of hemineglect. This variable was not related to lesion side and was thus given no further consideration.

\section{Discussion}

The results of this investigation strongly support the psychic tonus model -extending it to somesthesic perception in the parietal lobes. Unilateral parietal lesions typically cause a positive disturbance of body schema when they are located in the right hemisphere and a negative disturbance of body schema when they are located in the left hemisphere.

Why would a focal left parietal lesion, and only that lesion, impair a patient's finger gnosis (mental representation of the finger) at both hands as it does in Gerstmann's syndrome? Primary sensory somesthesic representation has been mapped in many species and has always been found to be fully contralateral and to present the same topography in each hemisphere in each posterolandic gyrus. None of the published reports on Gerstmann's syndrome has addressed the question of why both hands should manifest finger agnosia -except with perplexity [73] and when the bimanual nature of the agnosia is revealed, it is mentioned only incidentally or obliquely. The fact that autotopagnosia $[62,90,91]$ or Gerstmann's syndrome is so often due to a left hemisphere lesion has inspired several authors (e.g., [67, 74]) to state that body schema is left-hemisphere specialized - a thesis we categorically reject. Likewise, the fact that somatoparaphrenia can be due to a right sided lesion has led several other authors (e.g., [47, 92]) to conclude that body schema is right-hemisphere specialized - a thesis we also categorically reject. The laterality of lesions causing disorders of body schema is often acknowledged and stated to be perplexing by many authors not willing to commit themselves to a single hemispheric repository for body schema. Our psychic tonus model provides a clear explanation of the laterality of the lesion findings and the bilaterality of symptoms in the various syndromes of higher order 
body schema -and will hopefully put an end to some of these expressions of perplexity.

It seems to be a fact that when parietal neocortex is damaged unilaterally, control circuits of psychic tonus can become skewed, and a corresponding disorder of body schema can ensue, positive or negative, as a function of which hemisphere is damaged. Similar phenomena are observed for the other lobes and their respective functions: visual for the occipital lobes [8], auditory, sexual and paralinguistic for the temporal lobes [4,5,7,9], psychomotor for the frontal lobes [3]. These phenomena form an ensemble, a behavioral, motivational and attitudinal system which boils down to a certain type of energy expenditure/economy or approach/avoidance dynamic. Other metaphors of psychic tonus might include contrasts such as "upbeat/downtrodden" or "galavanised/inhibited". For the moment, our favorite designation of psychic tonus is to view it as a mechanism of energy management [1, 93]. In normal behavior, the interplay of these opposed hemispheric mechanisms is finely tuned to situational demands - though we suspect that it oscillates slowly (recall that the immune response is involved, and we expect that various hormones and neurotransmitters are involved as well). However, after a unilateral lesion, the patient becomes set in a mode which is no longer delicately adaptive.

The findings presented here demonstate that localized lesions can produce fractional effects on psychic tonus, such that only one representational modality may be affected - in the present case, somesthesic. Overall, the elements of psychic tonus tend to be correlated in normal and pathological conditions. Thus after a right hemisphere lesion, if a patient becomes manic, he/she (like any congenital manic) is also likely to be (but not necessarily) agitated, talkative, hypersexual, immunofacilitated, delusional and/or hallucinated. After a left hemisphere lesion, if a patient becomes depressed, he/she (like any congenital depressive) is likely to be (but not necessarily) lethargic, hypolalic, hyposexual, immunosuppressed and/or agnosic (see [1,3-5, 7-10] for reviews of the evidence).

It could be thought that the main mediator of the post lesion sticky switch is one or several neurotransmitters. Three good candidates are central norepinephrine serotonin and dopamine (all known to modulate functions subsumed under psychic tonus). Robinson and colleagues [94] ligated the middle cerebral artery of rats. Assays of brain catecholamines revealed 30 percent reductions of norepinephrine in the injured and uninjured cortex and locus coeruleus and a 20 percent reduction of dopamine in the substantia nigra in the right lesioned rats who had also become hyperactive. In contrast rats with left middle cerebral artery ligations did not become hyperactive and did not show any significant change in catecholamines in any of the brain areas studied. Similar asymmetry occurs in serotonin concentrations as a function of stroke side in humans [95]. It remains to be determined whether the latter effect is hemispherically symmetrical or not (a PET ligand study). In fact however we believe that such a neurotransmitter-mediated mechanism is not the principal determinant of hemispheric specialization for psychic tonus effects reported here and elsewhere. Indeed we have found that in non lesional epileptics with unilateral foci ictal hallucination is highly significantly more often observed in cases with left than right foci [96]. This corroboration of hemispheric specialization for psychic tonus cannot be explained as a chronic and major change of neurotransmitter concentration in the brain.

Dominant contemporary methodology in cognitive neuroscience has perhaps not been fully prepared to unveil such mechanisms. Indeed, fMRI cannot be expected (at least at present and without specific precautions) to reveal clearly and unequivocally lateralized (hemispherically specialized) processing, in the normal brain, of higher order body schema, and indeed it generally does not, for example when movement of one limb is required to be imagined [97,98]. Normal high level representation of the body (or of any other cognitive or perceptual function) comprises a balance of positive and negative representation, solliciting both hemispheres for the typical task, thus activating both hemispheres (but each in different aspects of the processing: for example one hemisphere might prepare the movement and the other might inhibit the contralateral mirror movement). A normally optimized high level representation, requiring imagination, polysensory integration, sensorimotor integration, etc., must balance dispositions toward too much imagination (overshooting, tangentiality, profuseness, fabulation, hallucination, etc.) against dispositions toward too much conservatism (inhibition, paucity, inattention, apathy, sluggishness, agnosia, etc.). A typical fMRI experiment subtracts one task from another in view of isolating a specific cognitive operation, thereby canceling out psychic tonus. Most importantly, metabolic imaging studies of cases of extreme distorsion of psychic tonus, specifically of body schema, do not form a corpus that can put a hemispheric specialization model to test. For intimate, subjective, complex experience such as the "body schema" the lesion method can provide insights 
for behavioral neuroscience where more expensive and prized methods such as metabolic brain imaging need to refine the tasks used in their protocols. With regard to body schema, one intriguing fMRI study required normal subjects to distinguish self movement from movement of another person. This task activated the right parietal lobe, but no theoretical explanation was provided for the laterality of the effect [99]. The "psychic tonus" model would interpret the task as a requirement for inhibition of body schema. Another intriguing fMRI study required normal subjects to localize their own body parts. This activated the left parietal lobe [89]. The "psychic tonus" model would construe this task as a requirement for "activation" of one's body schema. However, distinguishing other people's movement from our own might call upon "inhibition" of one's body schema.

\section{Acknowledgments}

This research was made possible by a grant from the Fonds de Recherche en Santé du Québec (FRSQ) to the first author and a bursary from the Natural Sciences and Engineering Research Council (NSERC) of Canada to the second author.

\section{References}

[1] C.M.J. Braun, The evolution of hemispheric specialization: A behavioral neurologic account and theorical model, Laterality (2006), in press.

[2] D. Galin, Implications for psychiatry of left and right cerebral specialization, Arch Gen Psychiat 31 (1974), 572-583.

[3] C.M.J. Braun, C. Larocque, S. Daigneault and I. MontourProulx, Mania, pseudomania, depression, and pseudodepression resulting from focal unilateral cortical lesions, Neuropsychiat Neuropsychol Behav Neurol 12 (1999), 35-51.

[4] C.M.J. Braun, M. Dumont, J. Duval, I. Hamel and L. Godbout, Opposed left and right brain hemisphere contributions to sexual drive: A multiple lesion case analysis, Behav Neurol 14 (2002), 55-61.

[5] C.M.J Braun, M. Dumont, J. Duval and I. Hamel, Speech rate as a sticky switch: A multiple lesion case analysis of mutism and hyperlalia, Brain Lang 89 (2004), 243-252.

[6] A. St-Marseille, E. Kouassi, G. D’Angelo, P. Laplante, S. Cheng, M.L. Trouvé, F. Gilbert, D. Geadah and C.M.J. Braun, Opposed left and right neocortical involvement in the immune response indexed by cerebrovascular accidents in humans: preliminary results, Brain Cogn 32 (1996), 173-175.

[7] C.M.J. Braun, J. Delisle and A. Guimond, Post lesion dysfunctional hyper and hypomnesia: Evidence for opposed hemispheric specialization, Cognitive Affective \& Behavioral Neuroscience, submitted 2007.
[8] C.M.J. Braun and A. Guimond, Visual hypo and hypergnosia as exemplars of poles of psychic tonus in the occipital lobes: Multiple case analyses, Cortex, submitted 2007.

[9] C.M.J. Braun, J. Duval and A. Guimond, Auditory hypergnosia as an example of psychic tonus in the temporal lobes: Multiple case analyses, Crit Rev Neurobiol 17 (2007), 145160.

[10] C.M.J. Braun, M. Dumont, J. Duval, I. Hamel-Hébert and L. Godbout, Brain modules of hallucination: A multiple lesion case analysis, J Psychiatr Neurosci 28 (2003), 432-449.

[11] O. Devinsky, Right cerebral hemisphere dominance for a sense of corporeal and emotional self, Epilepsy Behav 1 (2000), 60-73.

[12] G. Bottini, E. Bisiach, R. Sterzi and G. Vallarc, Feeling touches in someone else's hand, Neuroreport 13 (2002), 249-252.

[13] G. Denes, J.Y. Cappelletti, T. Zilli, F. Dalla Porta and A. Gallana, A category-specific deficit of spatial representation: the case of autotopagnosia, Neuropsychologia 38 (2000), 345350 .

[14] J. Schwoebel, H.B. Coslett and L.J. Buxbaum, Compensatory coding of body part location in autotopagnosia: Evidence for extrinsic egocentric coding, Cogn Neuropsychol 18 (2001), 363-381.

[15] M. Kinsbourne and H. Lempert, Human figure representation by blind children, J Gen Psychol 102 (1980), 33-37.

[16] J. Gerstmann, Problem of imperception of disease and of impaired body territories with organic lesions, Arch Neurol Psychiat 48 (1942), 890-913.

[17] J.A. Silva, G.B. Leong and A.L. Shaner, A classification system for misidentification syndromes, Psychopathology $\mathbf{2 3}$ (1990), 27-32.

[18] A. Pick, Storrung der orientierung am eigenen korper, Psychol Forsch 1 (1922), 303-318.

[19] H. Hecaen and M.L. Albert, Human Neuropsychology, Wiley, New York, 1978

[20] G. Gainotti, G. Miceli, M.C. Silveri and G. Villa, Some anatomo-clinical aspects of phonemic and semantic comprehension disorders in aphasia, Acta Neurol Scand 66 (1982), 652-665.

[21] A.S. Schwartz, P.L. Marchok, C.J. Kreinick and R.E. Flynn, The asymmetric lateralization of tactile extinction in patients with unilateral cerebral dysfunction, Brain 102 (1979), 669684.

[22] M. Jeannerod, Neurophysiological and neuropsychological aspects of spatial neglect, Advances in Psychology 45 (1987), North-Holland, Amsterdam.

[23] H.C. Haeske-Dewick, A.G. Canavan and V. Homberg, Directional hyperattention in tactile neglect within grasping space, J Clin Exp Neuropsychol 18 (1996), 724-732.

[24] E. Bors, Extinction and synesthesia in patients with spinal cord injuries, Paraplegia 17 (1979), 21-31.

[25] A. Donnet, A. Schmitt, M. Poncet, N. Graziani and F. Grisoli, Hallucinations de membres surnuméraires, héminégligence gauche et hypersexualité dans un cas d'hématome capsulolenticulaire droit, [Hallucinations of supernumerary limbs, left hemineglect and hypersexuality in a case of right capsulolenticular hematoma], Rev Neurol (Paris) 153 (1997), 587590.

[26] P.W. Halligan, J.C. Marshall and D.T. Wade, Unilateral somatoparaphrenia after right hemisphere stroke: a case description, Cortex 31 (1995), 173-182.

[27] G. Rode, N. Charles, M.T. Perenin, A. Vighetto, M. Trillet and G. Aimard, Partial remission of hemiplegia and somatopara- 
phrenia through vestibular stimulation in a case of unilateral neglect, Cortex 28 (1992), 203-208.

[28] A. Worthington and L. Beevers, Two arms, three hands: A supernumerary phantom phenomenon after right middle cerebral artery stroke, Neurocase 2 (1996), 135-140.

[29] J.A. Silva, J.L. Tekell, G.B. Leong and C.L. Bowden, Delusional misidentification of the self associated with nondominant cerebral pathology, J Clin Psychiat 56 (1995), 171.

[30] H. Foerstl, Capgras' delusion: an example of coalescent psychodynamic and organic factors, Compr Psychiatry 31 (1990), 447-449.

[31] D.L. Orsini, W.G. Van Gorp and K.B. Boone, The Neuropsychology Casebook, Springer-Verlag New York, Inc., New York, 1988.

[32] B.H. Price and M. Mesulam, Psychiatric manifestations of right hemisphere infarctions, J Nerv Ment Dis 173 (1985), 610-614.

[33] R. Ramasubbu, Conversion sensory symptoms associated with parietal lobe infarct: case report, diagnostic issues and brain mechanisms, J Psychiat Neurosci 27 (2002), 118-122.

[34] P. Vuilleumier, A. Reverdin and T. Landis, Four legs, Illusory reduplication of the lower limbs after bilateral parietal lobe damage, Arch Neurol 54 (1997), 1543-1547.

[35] R. Leiguarda, S. Starkstein, M. Nogues, M. Berthier and R. Arbelaiz, Paroxysmal alien hand syndrome, J Neurol Neurosur Ps 56 (1993), 788-792.

[36] M. Paulig, M. Weber and S. Garbelotto, Somatoparaphrenie Eine "plusvariante" der Anosognosie für Hemiplegie [Somatoparaphrenia. A "positive-variant" of anosognosia for hemiplegia], Nervenarzt 71 (2000), 123-129.

[37] J.K. Richardson, Psychotic behavior after right hemispheric cerebrovascular accident: A case report, Arch Phys Med Rehabil 73 (1992), 381-384.

[38] L. Maillard, J.P. Vignal, R. Anxionnat and L. Taillandier Vespignani, Semiologic value of ictal autoscopy, Epilepsia 45 (2004), 391-394.

[39] S. Nightingale, Somatoparaphrenia: A case report, Cortex 18 (1982), 463-467.

[40] M. Berthier and S. Starkstein, Acute atypical psychosis following a right hemisphere stroke, Acta Neurol Belg 87 (1987), 125-131.

[41] G. Assal, Non, je ne suis pas paralysée, c'est la main de mon mari, Schweiz Arch Neurol Neurochir Psychiatr 133 (1983), 151-157.

[42] E. Bisiach, M.L. Rusconi and G. Vallar, Remission of somatoparaphrenic delusion through vestibular stimulation, Neuropsychologia 29 (1991), 1029-1031.

[43] V.S. Ramachandran, The evolutionary biology of selfdeception, laughter, freaming and depression: Some clues from anosagnosia, Med Hypotheses 47 (1996), 347-362.

[44] E.A. Weinstein, R.L. Kahn, S. Malitz and J. Rozanski, Delusional reduplication of part of the body, Brain 77 (1954), 4560.

[45] F. Sellal, C. Renaseau-Leclerc and R. Labrecque, The man with 6 arms. An analysis of supernumerary phantom limbs after right hemisphere stroke, Rev Neurol (Paris) 152 (1996), 190-195.

[46] P.E.M. Carrilho, P. Caramelli, F. Cardoso, E. Reis Barbosa, C.A. Buchpiguel and R. Nitrini, Invonluntary hand levitation associated with parietal damage, Arq Neuro-psiquiatria 59 (2001), 521-525.

[47] S. Canavero, V. Bonicalzi, G. Castellano, P. Perozzo and B. Massa-Micon, Painful supernumerary phantom arm following motor cortex stimulation for central poststroke pain: Case report, J Neurosurg 91 (1999), 121-123.

[48] J. Fredericks, Occurence and nature of phantom limb phenomena following amputation of body parts and following lesions of the central and peripheral nervous system, Psychiatr Neurol, Neurochir 66 (1963), 73-97.

[49] V. Lunn, Autoscopic phenomena, Acta Psychiatr Scand 46 (1970), 118-125.

[50] I. Montour-Proulx, C.M.J. Braun, S.Daigneault, I. Rouleau, S. Kuehn, M. Piskopos and J. Bégin, Predictors of intellectual function after a unilateral cortical lesion: study of 635 patients from infancy to adulthood, J of Child Neurol 19 (2004), 935943.

[51] M. Baldini, L. Princi and P. Paoletti, Autotopoagnosia in angular gyrus neoplastic lesion: a case report, Acta Neurol (Napoli) 33 (1978), 451-455.

[52] A. Carota, M. Di Pietro, R. Ptak, D. Poglia and A. Schnider, Defective spatial imagery with pure Gerstmann's syndrome, Eur Neurol 52 (2004), 1-6.

[53] M. Gold, J.C. Adair, D.H. Jacobs and K.M. Heilman, Rightleft confusion in Gerstmann's syndrome: A model of body centered spatial orientation, Cortex 31 (1995), 267-283.

[54] M.D. Martory, E. Mayer, A.J. Pegna, J.M. Annoni, T. Landis and A. Khateb, Pure global acalculia following a left subangular lesion, Neurocase 9 (2003), 319-328.

[55] E. Mayer, M.D. Martory, A.J. Pegna, T. Landis, J. Delavelle and J.M. Annoni, A pure case of Gerstmann syndrome with a subangular lesion, Brain 122 (1999), 1107-1120.

[56] A. Ardila, M. Concha and M. Rosselli, Angular gyrus syndrome revisited: Acalculia, finger agnosia, right-left disorientation and semantic aphasia, Aphasiology 14 (2000), 743-754.

[57] D.N. Levine, R.B. Mani and R. Calvanio, Pure agraphia and Gerstmann's syndrome as a visuospatial-language dissociation: An experimental case study, Brain Lang 35 (1988), 172196.

[58] D.L. Sheimo, L.G. Bardach and P. Hilfinger, Fluent aphasia with Gerstmann's syndrome: A case study, Aphasiology 11 (1997), 283-291.

[59] M. Poncet, J.F. Pellissier, M. Sebahoun and C.J. Nasser, [Apropos of a case of autotopagnosia after a parieto-occipital lesion of the major hemisphere], Encéphale 60 (1971), 110-123.

[60] E. De Renzi and P. Faglioni, L'autotopoagnosia, Arch Psicol Neurol Psichiatr 24 (1963), 289-322.

[61] E. De Renzi and G. Scotti, Autotopagnosia: fiction or reality? Report of a case, Arch Neurol 23 (1970), 221-227.

[62] J.A. Ogden, Autotopagnosia, Occurrence in a patient without nominal aphasia and with an intact ability to point to parts of animals and objects, Brain 108 (1985), 1009-1022.

[63] P. Verstichel, J. Cambier, C. Masson, M. Masson and B. Robine, Apraxie et autotopoagnosie sans aphasie ni agraphie, mais avec activite compulsive de langage, au cours d'une lesion hemispherique droite [Apraxia and autotopoagnosia without aphasia or agraphia with compulsive language activity in right hemispheric lesion], Rev Neurol (Paris) 150 (1994), 274-281.

[64] C. Semenza, Impairment in localisation of body parts following brain damage, Cortex 24 (1988), 443-449.

[65] J.M. Nielsen, Agnosia, Apraxia, Aphasia: their Value in Cerebral Localization, Hafner Publishing Company: New York, 1946.

[66] N.R. Varney, Gerstmann syndrome without aphasia: a longitudinal study, Brain Cogn 3 (1984), 1-9.

[67] D.I. Arbuse, The Gerstmann syndrome, J Nerv Ment Dis 105 (1947), 359-371. 
[68] F.J. Perez Blanco, A. Rodriguez Cuartero, F. Morata Garcia de la Puerta and L. Perez Costillas, Complete Gerstmann's syndrome, Rev Clin Esp 185 (1989), 221-222.

[69] J. M. Calvo-Romero, J.C. Bureo-Dacal and J.L. RamosSalado, Gerstmann syndrome caused by right parietal ischemic stroke, Rev Neurol 30 (2000), 398-399.

[70] S. Maeshima, T. Kuwata, O. Masuo, H. Yamaga, R. Okita, F. Ozaki, H. Moriwaki and P. Roger, Transcortical sensory aphasia due to a left frontal subcortical haemorrhage, Brain Inj 13 (1999), 927-933.

[71] M.R. Moore, J.L. Saver, K.A. Johnson and J.A. Romero, Right parietal stroke with Gerstmann's syndrome, Arch Neurol 48 (1991), 432-435.

[72] K. Dozono, K. Hachisuka, A. Ohnishi and H. Ogata, Gerstmann's syndrome and ideational apraxia with a right cerebral hemispheric lesion: a case report, Neurocase 3 (1997), 61-66.

[73] W.L. Sobota, W.H. Restum and E. Rivera, A case report of Gerstmann's syndrome without aphasia, Int J of Clin $\mathrm{Neu}$ ropsyc 7 (1985), 157-164

[74] M. Mazzoni, L. Pardossi, R. Cantini, V. Giorgetti and R. Arena, 1990. Gerstmann syndrome: a case report, Cortex 26 (1990), 459-467.

[75] E. Mayer, M.D. Martory, A.J. Pegna, T. Landis, J. Delavelle and J.M. Annoni, A pure case of Gerstmann syndrome with a subangular lesion, Brain 122 (1999), 1107-1120.

[76] O. Tucha, A. Steup, C. Smely and K.W. Lange, Toe agnosia in Gerstmann syndrome, J Neurol Neurosur Psy 63 (1997), 399-403.

[77] A. Ohtagaki, Y. Maeoka, M. Shiota and T. Koeda, A case of learning-disabled child with a white matter lesion in the left parietal lobe, No To Hattatsu 30 (1998), 69-74.

[78] B.Z. Garty, Y. Waisman and R. Weitz, Gerstmann tetrad in leopard syndrome, Pediatr Neurol 5 (1989), 391-392.

[79] D.P. Roeltgen, S. Sevush and K.M. Heilman, Pure Gerstmann's syndrome from a focal lesion, Arch Neurol 40 (1983), 46-47.

[80] M. Kinsbourne and D.B. Rosenfield, Agraphia selective for written spelling, Brain Lang 1 (1974), 215-225.

[81] M. Kinsbourne and E.K. Warrington, A study of finger agnosia, Brain 85 (1962), 47-66.

[82] C. Fournier del Castillo, J.J. Garcia-Penas, L.G. GutierrezSolana and M.L. Ruiz-Falco Rojas, Gerstmann's syndrome in a 9 year old boy, Rev Neurol 30 (2000), 731-736.

[83] M. Trillet, B. Croisile and B. Laurent, L'agraphie pure: à propos de deux cas [Pure agraphia. About two cases.], Rev Neurol (Paris) 145 (1989), 720-724.

[84] S. Dehaene and L. Cohen, Cerebral pathways for calculation: double dissociation between rote verbal and quantitative knowledge of arithmetic, Cortex 33 (1997), 219-250.
[85] S.H. Lisanby, C. Kohler, C.L. Swanson, and R.E. Gur, Psychosis Secondary to Brain Tumor, Sem Clin Neuropsychiatr 3 (1998), 12-22.

[86] S.S. Shergilla, L.A. Camerona, M.J. Brammerb, S.C.R. Williamsc, R.M. Murrayaand and P.K. McGuire, Modality specific neural correlates of auditory and somatic hallucinations, J Neurol Neurosurg Psychiatr 71 (2001), 688-690.

[87] I. McGilchrist and J. Cutting, Somatic delusions in schizophrenia and the affective psychoses, Brit J Psychiatr 167 (1995), 350-361.

[88] J. Hutsler and R.A. Galuske, Hemispheric asymmetries in cerebral cortical networks, Trends Neurosci 26 (2003), 429435.

[89] E. Vanroose, M. Marchau, I. Dehaene and M. Lammens, (Altitudinal hemianopia; a clinical and anatomical entity or a mere coincidence? Case report and review of literature, Acta Neurol Belgica 90 (1990), 254-264.

[90] O. Felician, M. Ceccaldi, M. Didic, C. Thinus-Blanc and M. Poncet, Pointing to body parts: a double dissociation study, Neuropsychologia 41 (2003), 1307-1316.

[91] C. Guariglia, L. Piccardi, M.C. Puglisi Allegra and M. Traballesi, Is autotopagnosia real? EC says yes, A case study, Neuropsychologia 40 (2002), 1744-1749.

[92] A.A. Naga, O. Devinsky and W.B. Barr, Somatoform disorders after temporal lobectomy, Cogn Behav Neurol 7 (2004), 5761.

[93] A.D. Craig, Forebrain emotional asymmetry: a neuroanatomical basis? Trends Cogn Sci 9 (2005), 566-571.

[94] R.G. Robinson, Differential behavioral and biochemical effects of right and left hemispheric cerebral infarction in the rat, Science 205 (1979), 707-710.

[95] R.G. Robinson and S.E. Starkstein, Mood disorders following stroke: new findings and future directions, J Ger Psychiat 22 (1989), 1-15

[96] A. Guimond, Visual auditory and somesthesic paroxystic hypergnosia as examples of psychic tonus: Multiple case analyses, Master's thesis, Universite du Quebec à Montreal, 2007.

[97] E. Bonda, M. Petrides, S. Frey and A. Evans, Neural correlates of mental transformations of the body-in-space, Proc Natl Acad Sci USA 92 (1995), 11180-11184.

[98] P.E. Roland, B. Larsen, N.A. Lassen and E. Skinhoj, Supplementary motor area and other cortical areas in organization of voluntary movements in man, J Neurophysiol 43 (1980), 118-136.

[99] D.T. Leube, G. Knoblich, M. Erb and T.T. Kircher, Observing one's hand become anarchic: an fMRI study of action identification, Conscious Cogn 12 (2003), 597-608. 


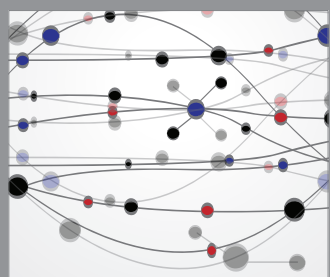

The Scientific World Journal
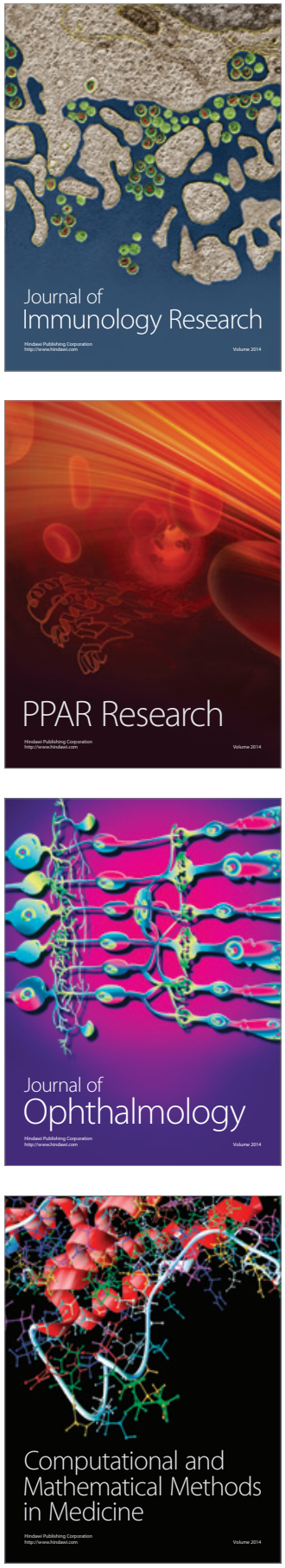

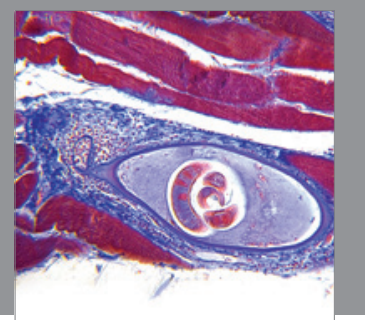

Gastroenterology

Research and Practice
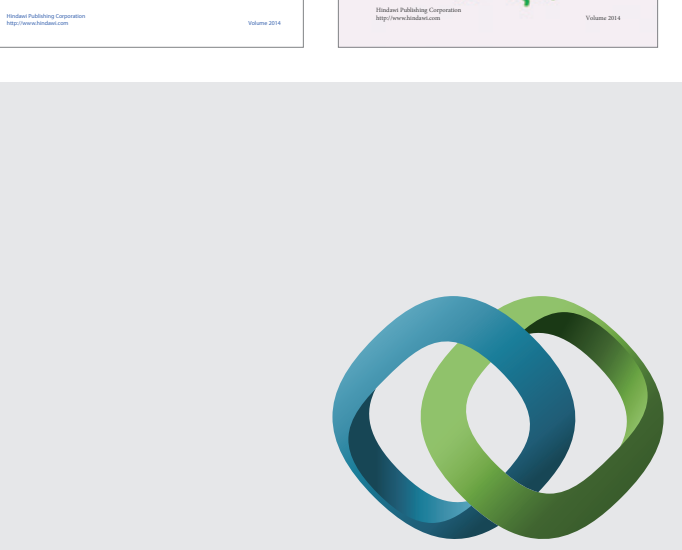

\section{Hindawi}

Submit your manuscripts at

http://www.hindawi.com
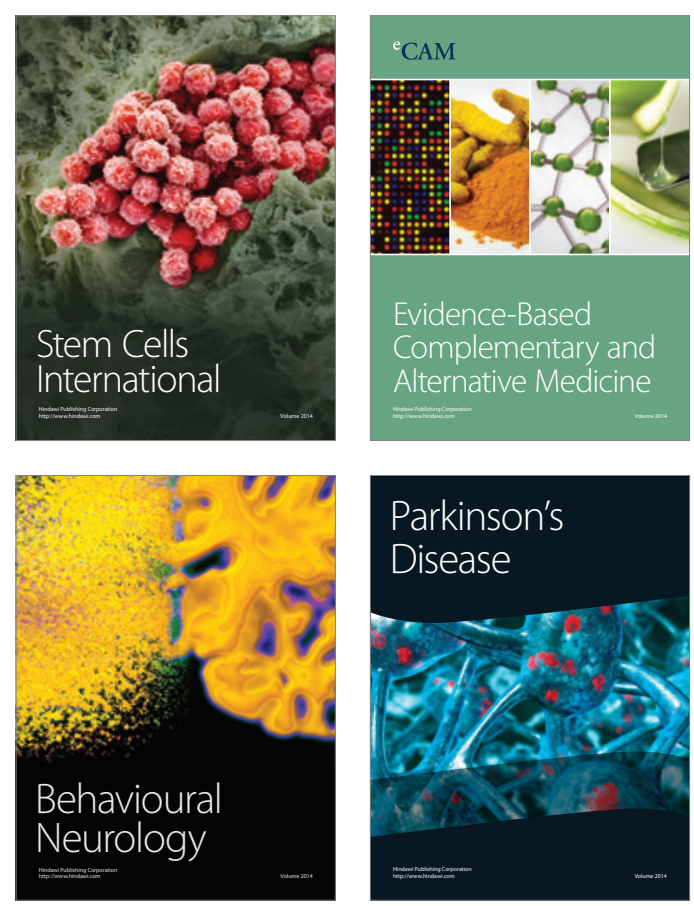

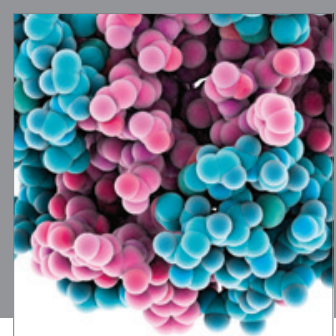

Journal of
Diabetes Research

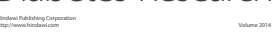

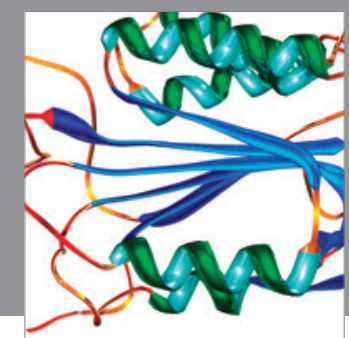

Disease Markers
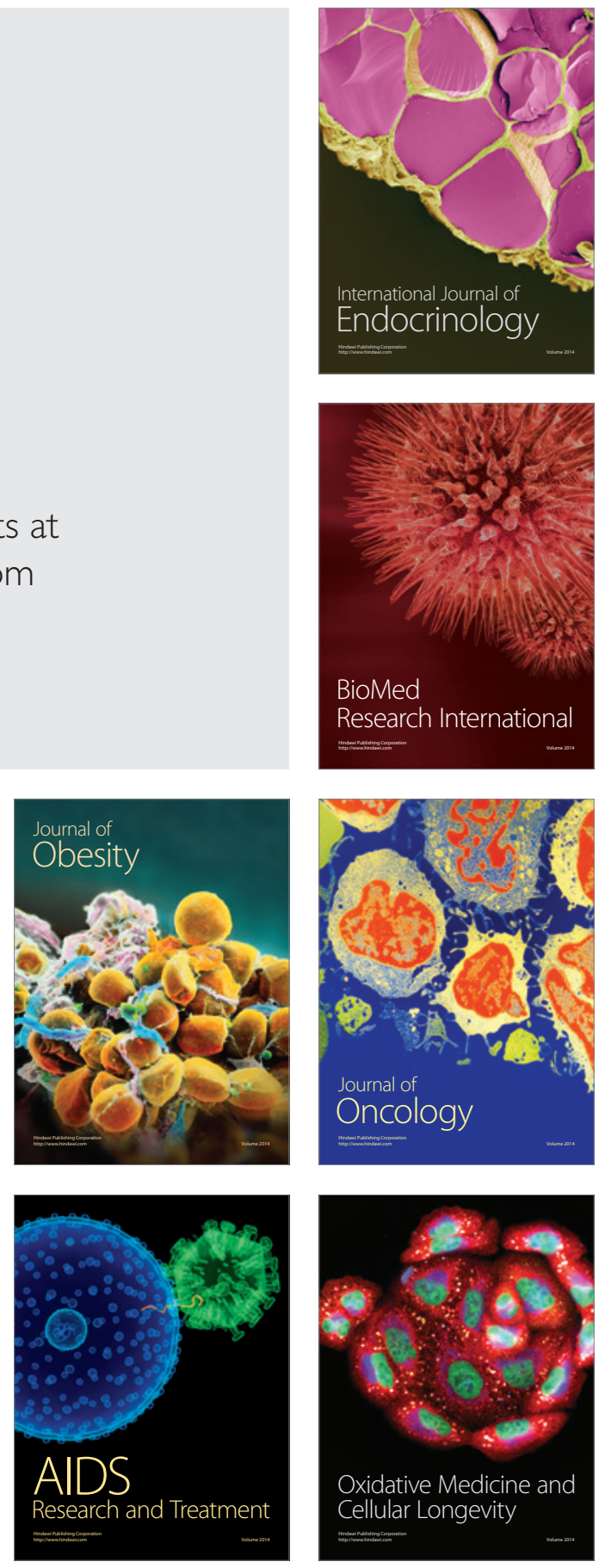